\title{
Structural-Identification Aspects of Decision-Making in Systems with Bouc-Wen Hysteresis
}

\author{
Nikolay Karabutov \\ Department of Control Problems, MIREARussian Technological University, Moscow, Russia \\ Email: kn22@yandex.ru, nik.karabutov@gmail.com
}

How to cite this paper: Karabutov, N. (2021) Structural-Identification Aspects of Decision-Making in Systems with Bouc-Wen Hysteresis. Intelligent Control and Automation, 12, 91-118. https://doi.org/10.4236/ica.2021.124006

Received: December 27, 2021

Accepted: February 6, 2022

Published: February 9, 2022

Copyright $\odot 2021$ by author(s) and Scientific Research Publishing Inc. This work is licensed under the Creative Commons Attribution International License (CC BY 4.0).

http://creativecommons.org/licenses/by/4.0/

\section{(c) (i) Open Access}

\begin{abstract}
Considering the structural analysis problem of systems properties with BoucWen hysteresis $(\mathrm{BWH})$, various approaches are proposed for the identification of BWH parameters. The applied methods and algorithms are based on the design of parametric models and consider a priori information and the results of data analysis. Structural changes in the BWH form a priori. Methods for the Bouc-Wen model (BWM) identification and its structure estimation are not considered under uncertainty. The study's purpose is the analysis the structural problems of the Bouc-Wen hysteresis identification. The analysis base is the application of geometric frameworks (GF) under uncertainty. Methods for adaptive estimation parameters and structural of BWM were proposed. The adaptive system stability is proved based on vector Lyapunov functions. An approach is proposed to estimate the identifiability and structure of the system with BWH. The method for estimating the identifiability degree based on the analysis of GF is considered. BWM modifications are proposed to guarantee the system's stability and simplify its description.
\end{abstract}

\section{Keywords}

Structure, Framework, Identification, Structural Identifiability, Bouc-Wen Hysteresis, Nonlinearity, Adaptation

\section{Introduction}

Various models are used to describe hysteresis [1]. The Bouc-Wen model (BWM) is widely used to describe hysteresis. BWM is proposed by R. Bouc [2] and is generalized by Y.K. Wen [3] (system $S_{B W}$ )

$$
m \ddot{x}+c \dot{x}+F(x, z, t)=f(t),
$$




$$
\begin{gathered}
F(x, z, t)=\alpha k x(t)+(1-\alpha) k d z(t), \\
\dot{z}=d^{-1}\left(a \dot{x}-\beta|\dot{x}||z|^{n} \operatorname{sign}(z)-\gamma \dot{x}|z|^{n}\right),
\end{gathered}
$$

where $m>0$ is mass, $c>0$ is damping, $F(x, z, t)$ is the recovering force, $d>0, n>0, k>0, \alpha \in(0,1), f(t)$ is exciting force, $a, \beta, \gamma$ are some numbers. Equation (3) is the BWM.

Many modifications of BWM [4] are proposed. Each model considers the features of the considered object. The BWM successful application depends on the identification of its parameters. The solution of the nonlinear Equation (3) is the main problem of BWM identification. The methods of identification and control Bouc-Wen hysteresis systems are given in [5] [6]. Adaptive modelling methods [6] are used to analyze the state of structural dynamics objects. An approach to the BWH identification based on analysis of a priori information and some heuristic procedures is proposed in [7]. Adaptive algorithms are proposed in [8] [9] for the BWM parameters estimation with the data forgetting [10]. It is assumed that are available for measurement $\ddot{x}$ and $z$, and $\dot{x}, x$ are obtained by integration. The approach to adaptive identification [11] [12] is based on the least-squares method application and correction of the gain matrix. Change areas $\dot{x}, x$ and the parameter $n$ value are set. The adaptive observers use for the BWH identification is considered in [13]. The analysis of other approaches to the BWH parameters identification is given in [4] [14] [15] [16] [17]. Most procedures are based on measuring derivatives $x$. This possibility does not always exist when solving practical problems.

Examples [18] are known when BWM parameters estimations do not coincide with results obtained for other inputs. Such examples speak about the ambiguity of identification, which causes the instability of the model. Explain it with the fact that the Bouc-Wen model should be stable and ensure the adequacy of a physical process [19]. Requirements for BWM [19]: 1) adequacy of the mathematical model to the physical process; 2) BWM stability. Stability conditions impose restrictions on the changing area of model parameters. The choice of parameters belonging to the stability domain does not always give the adequate BVM [16] under uncertainty. Therefore, the approach [18] to the hysteresis; 3) parameters identification based on the BWH approximation of polynomial is proposed.

So, the analysis of publications shows that many algorithms and procedures for the BWM parameters identification propose. Proposed models consider features of the system. As a rule, the area of the BWM parameters changing sets a priori. Some parameters, such as $n$, are considered known. It is often assumed that derivatives of the system are measured. This situation does not always occur in practice and gives to the non-realizability of algorithms. The structure choice of the system (1)-(3) (structural identification (SI)) is the use result of the researcher's knowledge and intuition. This approach does not always give an adequate choice of the BWM structure under uncertainty. Often the structural iden- 
tification problem is reduced to the parametric identification problem [20] [21]. This approach is laborious under uncertainty.

So, we see that BWH structural identification problem has not been developed. The $S_{B W}$-system modeling effectiveness depends on the choice of its parameters due to its initial instability. The input perturbation choice is significant for obtaining adequate results. The incorrect choice of input can lead to a system's non-identifiability. These problems require a solution for BWH. Some results on these problems are presented in [22].

The systematic approach proposed in this work gives the problem solve of identification systems with Bouc-Wen hysteresis. It includes: 1) the method for the input affect estimation on the $S_{B W}$-system identifiability; 2) a hierarchical immersion method that allows you to decide on the BWH structure under uncertainty; 3) the adaptive identification of BWH parameters based on input-output data; 4) the method for estimating the identifiability degree based on the analysis of GF and the phase portrait of the $S_{B W}$-system.

Work structure: 1) problem statement; 2) the method of $S_{B W}$-system adaptive identification; 3) modifications of the $S_{B W}$ system to simplify it and ensure stability; 4) the estimation method of structural identification and identifiability $S_{B W}$-systems; 5) the properties analysis of the input $f(t)$, which guarantees the SI and identifiability of the $S_{B W}$-system; 6) BMW modifications guarantee its stability.

Remark 1. The parametric approach does not allow estimating of the BWH structure under uncertainty. The proposed approach is based on the properties analysis of geometric frameworks.

\section{Problem Statement}

Consider $S_{B W}$-system. We have information on the input and the output

$$
\mathrm{I}_{o}=\left\{f(t), x(t), t \in\left[t_{0}, t_{e}\right]\right\},
$$

where $t_{e}<\infty, f(t), x(t)$ are limited functions of time.

Determine conditions of the system $S_{B W}$ identifiability and structural components of the Bouc-Wen model (3) based on analysis of the sets (4).

Solving this problem is answered to the question: can we get an estimate of the system (3) structure under uncertainty?

Consider the identification of system parameters (1)-(3) by $\mathrm{I}_{o}$.

\section{Adaptive Identification of BWH}

\subsection{Problem Statement}

Consider the system $S_{B W}$. Let $y=x$ be the output of the system. The set of the experimental data is $\mathrm{I}_{o}=\{f(t), y(t), t \in J\}$, where $J \subset R$ is the specified time interval.

Designate by the parameters vector of the system as $A=[m, c, a, k, \alpha, \beta, \gamma, n]^{\mathrm{T}}$.

Problem: an adaptive observer to design for the evaluation of vector $A$ such 
that

$$
\lim _{t \rightarrow \infty}|\hat{y}(t)-y(t)| \leq \pi_{y},
$$

where $\hat{y} \in R$ is the output of the adaptive observer, $\pi_{y} \geq 0$.

Remark 2. The identification effectiveness of the system $S_{B W}$ depends on features of the input $f(t)$. Requirements to $f(t)$ in identification problems are known. The force $f(t)$ must satisfy the constant excitation (CE) condition. This condition is necessary but not enough [23]. The input having the CE property cannot ensure the identifiability of the hysteresis structure. The structural identifiability of hysteresis is possible if $f(t)$ has the S-stabilization property of the system [23].

\subsection{Adaptive System Identification}

The set $I_{o}$ has the form (4). Therefore, it is not applicable for estimating the parameters of the system $S_{B W}$. Design an adaptive observer for the system (1)-(3).

Consider the simplified system (1)-(3) when $d=1, a=1$. Substitute $F(x, z, t)$ in (1), and divide it by $s+\mu$, where $\mu>0$ does not coincide with roots of the polynomial $s^{2}+a_{1} s+a_{2}, s=d / d t$. Then

$$
\begin{aligned}
\dot{x} & =a_{1} x+a_{2} p_{x}+a_{3} p_{z}+b p_{f}, \\
\dot{p}_{x} & =-\mu p_{x}+x, \dot{p}_{f}=-\mu p_{f}+f, \\
\dot{p}_{z} & =-\mu p_{z}+z,
\end{aligned}
$$

where $a_{1}=-(c-\mu m) / m, \quad a_{2}=-(\alpha k-\mu(c-\mu m)) / m, \quad a_{3}=-(1-\alpha) k / m$.

Equations (6), (7) contain only measurable variables except $z$. It complicates the identification process of the system $S_{B W}$ parameters. Apply the model

$$
\dot{\hat{x}}=-k_{x}(\hat{x}-x)+\hat{a}_{1} x+\hat{a}_{2} p_{x}+\hat{a}_{3} p_{z}+\hat{b} p_{f}
$$

to the estimation of the system (6) parameters, where $k_{x}>0$ is the specified number; $\hat{a}_{i}(t), i=1,2,3$ and $\hat{b}(t)$ are adjusted parameters.

Designate $e=\hat{x}-x$. Obtain the equation for the identification error from (6), (8)

$$
\dot{e}=-k_{x} e+\Delta a_{1} x+\Delta a_{2} p_{x}+\Delta a_{3} p_{z}+\Delta b p_{f},
$$

where $\Delta b=\hat{b}(t)-b, \Delta a_{1}=\hat{a}_{1}(t)-a_{1}, \Delta a_{2}=\hat{a}_{2}(t)-a_{2}, \Delta a_{3}=\hat{a}_{3}(t)-a_{3}$.

(9) is not solvable as the variable $z$ is unknown in (7). Obtain the current estimation for $z(t)$. Consider model

$$
\dot{\hat{x}}_{\overline{\bar{z}}}=-k_{x}\left(\hat{x}_{\bar{z}}-x\right)+\hat{a}_{1} x+\hat{a}_{2} p_{x}+\hat{b} p_{f} .
$$

Determine the misalignment $\varepsilon_{z}=x-\hat{x}_{\bar{z}}$ and use it for the variable $z$ estimation. Let $\varepsilon_{z}$ is the current estimation $z$. Apply the model to the estimation $z$

$$
\dot{\hat{z}}=-k_{z}\left(\hat{z}-\varepsilon_{z}\right)+\tilde{\dot{X}}-\hat{\beta}|\tilde{\dot{x}}||\hat{z}|^{n} \operatorname{sign}(\hat{z})-\hat{\gamma} \tilde{\dot{x}}|\hat{z}|^{n},
$$

where $\tilde{\dot{x}}=(x(t+\tau)-x(t)) / \tau ; k_{z}>0$ is the given number $\hat{\beta}, \hat{\gamma}$ are the hysteresis (3) parameters estimations; $\tau$ is the integration step. 
Introduce the misalignment $\varepsilon=\hat{z}-\varepsilon_{z}$ and obtain the equation for $\varepsilon$

$$
\begin{aligned}
& \dot{\varepsilon}=-k_{z} \varepsilon+\Delta \dot{x}+\Delta \beta|\tilde{\dot{x}}||\hat{z}|^{n} \operatorname{sign}(\hat{z})+\beta \eta_{\beta}+\Delta \gamma \tilde{\dot{x}}|\hat{z}|^{n}+\gamma \eta_{\gamma}, \\
& \eta_{\beta}=|\dot{x}||z|^{n} \operatorname{sign}(z)-|\tilde{x}||\hat{z}|^{n} \operatorname{sign}(\hat{z}), \quad \eta_{\gamma}=\dot{x}|z|^{n}-\tilde{\dot{x}}|\hat{z}|^{n},
\end{aligned}
$$

where $\Delta \dot{x}=\tilde{\dot{x}}-\dot{x}, \Delta \beta=\beta-\hat{\beta}, \Delta \gamma=\gamma-\hat{\gamma}$.

Present (8) as

$$
\dot{\hat{x}}=-k_{x}(\hat{x}-x)+\hat{a}_{1} x+\hat{a}_{2} p_{x}+\hat{a}_{3} p_{\hat{z}}+\hat{b} p_{f},
$$

where

$$
\dot{p}_{\hat{z}}=-\mu p_{\hat{z}}+\hat{z} .
$$

Then (9) rewrite as

$$
\dot{e}=-k_{x} e+\Delta a_{1} x+\Delta a_{2} p_{x}+\Delta a_{3} p_{\hat{z}}+\Delta b p_{f},
$$

and adaptive algorithms describe as

$$
\Delta \dot{a}_{1}=-\gamma_{1} e x, \Delta \dot{a}_{2}=-\gamma_{2} e_{x}, \Delta \dot{a}_{3}=-\gamma_{3} e p_{\hat{z}}, \Delta b=-\gamma_{b} e p_{f},
$$

where $\gamma_{i}>0, i=1,2,3 ; \gamma_{b}>0$.

Tuning algorithms for $\Delta \beta$ and $\Delta \gamma$ in (11) have the form

$$
\begin{aligned}
& \Delta \dot{\beta}=-\chi_{\beta} \varepsilon|\tilde{\dot{x}}||\hat{z}|^{n} \operatorname{sign}(\hat{z}), \\
& \Delta \dot{\gamma}=-\chi_{\gamma} \varepsilon \tilde{\dot{X}}|\hat{z}|^{n},
\end{aligned}
$$

where $\chi_{\beta}>0, \chi_{\gamma}>0$ are parameters ensuring a convergence of algorithms.

Several algorithms are applicable for the indicator $n$ estimation in (11). The effectiveness of their work depends on several factors. The simple algorithm has the form

$$
\dot{\hat{n}}= \begin{cases}-\gamma_{n} \varepsilon \hat{\beta}|\hat{z}|^{\hat{n}-1} \hat{z} \tilde{\dot{x}}, & \text { if }\left|\frac{\varepsilon}{\varepsilon_{z}}\right| \in\left[v_{0}, v_{1}\right], \\ 0, & \text { if }\left|\frac{\varepsilon}{\varepsilon_{z}}\right| \notin\left[v_{0}, v_{1}\right],\end{cases}
$$

where $v_{0}, v_{1}$ are set positive numbers, $\gamma_{n}>0$.

Remark 3. The identification procedure stability is the main problem of the system synthesis with BWM. We propose the method based on adaptive observer application.

\subsection{Properties of Adaptive System}

We estimate the adaptive system stability by applying Lyapunov vector functions. Consider the subsystem $A S_{X}$ described by (14), (15). Let

$$
\begin{gathered}
\Delta K(t) \triangleq\left[\Delta a_{1}(t), \Delta a_{2}(t), \Delta a_{3}(t), \Delta b(t)\right]^{\mathrm{T}}, \\
V_{K}(t) \triangleq 0.5 \Delta K^{\mathrm{T}}(t) \Gamma^{-1} \Delta K(t), \\
V(t)=V_{e}(t)+V_{K}(t),
\end{gathered}
$$

where $\Gamma=\operatorname{diag}\left(\gamma_{1}, \gamma_{2}, \gamma_{3}, \gamma_{b}\right)$. Next, we give a results generalization [23] [24]. 
Assumption 1. The input $f(t)$ is constantly exciting and limited.

Theorem 1. Let 1) functions (19), $V_{K}(t)$ are positive definite and satisfy conditions $\left.\inf _{|e| \rightarrow \infty} V_{e}(e) \rightarrow \infty, \inf _{\|\Delta K\| \rightarrow \infty} V_{K}(\Delta K) \rightarrow \infty ; 2\right)$ assumption 1 for the system (1)-(3) is satisfied. Then all trajectories of the system $A S_{X}$ are limited belong area $\mathrm{G}_{t}=\left\{(e, \Delta K): V(t) \leq V\left(t_{0}\right)\right\}$ and the estimation $\int_{t_{0}}^{t} 2 k_{x} V_{e}(\tau) \mathrm{d} \tau \leq V\left(t_{0}\right)-V(t)$ is fair.

Theorem 1 shows the limitation of adaptive system trajectories. The asymptotical stability ensuring the system demands to impose additional conditions.

Let $P(t) \triangleq\left[x(t) p_{x}(t) p_{\hat{z}}(t) p_{f}(t)\right]^{T}$.

Definition 1 . The vector $P$ is constantly excited with a level $v$ or have property $P E_{v}$ if $P E_{v}: P(t) P^{\mathrm{T}}(t) \geq v I_{4}$ fairly for $\exists v>0$ and $\forall t \geq t_{0}$ on some interval $T>0$, where $I_{4} \in R^{4}$ is the unity matrix.

If the vector $P(t)$ has property $\mathscr{P} E_{v}$ then we will write $P(t) \in \mathscr{P} E_{v}$.

The system $S_{B W}$ is stable, and the input $f(t)$ is restricted. Therefore, present the property $P E_{v}$ for the matrix $B_{P}(t)=P(t) P^{\mathrm{T}}(t)$ as

$$
\mathcal{P} E_{v, \bar{v}}: v I_{4} \leq B_{P}(t) \leq \bar{v} I_{4}, \forall t \geq t_{0},
$$

where $\bar{v}>0$ is some number.

Let the estimation to $V_{K}(t)$ be fair

$$
0.5 \beta_{l}^{-1}(\Gamma)\|\Delta K(t)\|^{2} \leq V_{K}(t) \leq 0.5 \beta_{1}^{-1}(\Gamma)\|\Delta K(t)\|^{2},
$$

where $\beta_{1}(\Gamma), \beta_{l}(\Gamma)$ are minimum and maximum eigenvalues of the matrix $\Gamma$.

Apply inequalities (20), (21) and obtain estimations for $\dot{V}_{e}, \dot{V}_{K}$

$$
\begin{gathered}
\dot{V}_{e} \leq-k_{x} V_{e}+\frac{\bar{v} \beta_{l}(\Gamma)}{k_{x}} V_{K}, \\
\dot{V}_{K} \leq-\frac{3}{4} \vartheta v \beta_{1}(\Gamma) V_{K}+\frac{8}{3} \vartheta V_{e},
\end{gathered}
$$

Theorem 2. Let conditions be satisfied 1) positive definite Lyapunov functions $V_{e}(t)$ and (18) allow the indefinitely small highest limit at $|e(t)| \rightarrow 0$, $\|\Delta K(t)\| \rightarrow 0$; 2) $P(t) \in P E_{v, \bar{v}}$; 3) equality $e \Delta K^{\mathrm{T}} P=\vartheta\left(\Delta K^{\mathrm{T}} B \Delta K+e^{2}\right)$ is fair in the area $O_{v}(O)$ with $0<\vartheta$, where $O=\left\{0,0^{3 m}\right\} \subset R \times R^{3 m} \times J_{0, \infty}, O_{v}$ is some neighborhood of the point $O$; 4) the function $V_{K}(t)$ satisfies $\left.(21) ; 5\right)$ $\dot{V}_{\varepsilon}, \dot{V}_{K}$ satisfy the system of inequalities

$$
\left[\begin{array}{c}
\dot{V}_{e} \\
\dot{V}_{K}
\end{array}\right] \leq \underbrace{\left[\begin{array}{cc}
-k_{x} & \frac{\bar{v} \beta_{l}(\Gamma)}{k_{x}} \\
\frac{8}{3} \vartheta & -\frac{3 v \vartheta \beta_{1}(\Gamma)}{4}
\end{array}\right]}_{A_{V}}\left[\begin{array}{c}
V_{e} \\
V_{K}
\end{array}\right] ;
$$

6) the upper solution for $V_{e, K}(t)=\left[V_{e}(t) V_{K}(t)\right]^{\mathrm{T}}$ satisfies to the comparison equation $\dot{S}=A_{v} S$ if 


$$
V_{\rho}(t) \leq s_{\rho}(t), \forall\left(t \geq t_{0}\right) \&\left(V_{\rho}\left(t_{0}\right) \leq s_{\rho}\left(t_{0}\right)\right)
$$

where $\rho=e, K, S=\left[s_{e} s_{K}\right]^{\mathrm{T}}, A_{V} \in R^{2 \times 2}$ is $M$-matrix. Then the system $A S_{X}$ is exponentially stable with the estimation

$$
V_{e, K}(t) \leq \mathrm{e}^{A_{V}\left(t-t_{0}\right)} S\left(t_{0}\right),
$$

if

$$
k_{x} \geq \frac{4}{3} \sqrt{\frac{2 \bar{v} \beta_{l}(\Gamma)}{v \beta_{1}(\Gamma)}} .
$$

Theorem 2 shows that the adaptive system gives the estimates for system (1) parameters. It is fair at the fulfillment of conditions (27). We supposed that the variable $p_{\hat{z}}$ restricted. The boundedness of the variable $\hat{X}_{\bar{z}}$ follows from the boundedness of the system $A S_{X}$ trajectories.

Consider subsystem $A S_{Z}$ described by Equations (12), (16). Introduce Lyapunov functions

$$
\begin{aligned}
& V_{\varepsilon \beta \gamma}(t)=V_{\varepsilon}(t)+V_{\beta, \gamma}(t), \\
& V_{\beta, \gamma}(t)=0.5 \chi_{\beta}^{-1}(\Delta \beta(t))^{2}+0.5 \chi_{\gamma}^{-1}(\Delta \gamma(t))^{2} .
\end{aligned}
$$

Theorem 3. Let 1) functions $V_{\varepsilon}(t), V_{\beta, \gamma}(t)$ are positive definite and satisfy condition

$$
\inf _{|\varepsilon| \rightarrow \infty} V_{\varepsilon}(\varepsilon) \rightarrow \infty, \inf _{\|[\Delta \beta, \Delta \gamma]\| \rightarrow \infty} V_{\beta, \gamma}(\Delta \beta, \Delta \gamma) \rightarrow \infty ;
$$

2) the function $V_{\varepsilon \beta \gamma}(t)$ has the form $\left.(28) ; 3\right)$ the function

$$
\tilde{g}_{1}(t)=\sup _{\varepsilon \in \Omega} \frac{|\varepsilon|^{n+1}(t)}{V_{\varepsilon}(t, \varepsilon)}, \quad g_{1}=\sup _{\varepsilon \in \Omega} \tilde{g}_{1}(t) \text {, }
$$

exists, where $\Omega$ is the definition range of the subsystem $\left.A S_{Z} ; 4\right)|\Delta \dot{x}| \leq \delta_{\Delta}$, $\left.\left.\delta_{\Delta} \geq 0 ; 5\right) \quad|\dot{x}| \leq v, v>0 ; 6\right)$ the assumption 1 holds for the system (1)-(3). Then all trajectories of the system $A S_{Z}$ are bounded, belong in the area $\mathrm{G}_{\varepsilon}=\left\{(\varepsilon, \Delta \beta, \Delta \gamma): V_{\varepsilon \beta \gamma}(t) \leq V_{\varepsilon \beta \gamma}\left(t_{0}\right)\right\}$, and the estimation

$$
\begin{aligned}
& \int_{t_{0}}^{t}\left(k_{z}-v(\beta+\gamma) g_{1}\right) V_{\varepsilon}(\tau) \mathrm{d} \tau+\frac{1}{2\left(k_{z}-v(\beta+\gamma) g_{1}\right)\left(t-t_{0}\right)}\left(\delta_{\Delta}\right)^{2} \\
& \leq V_{\varepsilon \beta \gamma}\left(t_{0}\right)-V_{\varepsilon \beta \gamma}(t)
\end{aligned}
$$

is fair if

$$
k_{z}>v(\beta+\gamma) g_{1} .
$$

So, the boundedness of trajectories in the adaptive system is proved. The analysis showed that the subsystem $A S_{X}$ is asymptotically stable. The prove of trajectories boundedness for the subsystem $A S_{Z}$ is a more complex problem. The estimation (31) shows that the quality of processes in the $A S_{Z}$-system depends on the output derivative of the $S_{B W}$-system. The following result give more exact estimations for processes in the $A S_{Z}$-system.

Theorem 4. Let 1) positive definite Lyapunov functions $V_{\beta, \gamma}(t)$ and $V_{\varepsilon}(t)$ 
exist and have the indefinitely small higher limit at $\|[\Delta \beta(t), \Delta \gamma(t)]\| \rightarrow 0$ to $\left.|\varepsilon(t)| \rightarrow 0 ; 2) \quad P(t) \in P E_{V, \bar{v}} ; 3\right)$ such $c_{1}>0, c_{2}>0$ exist that conditions

$$
\begin{aligned}
& \varepsilon \Delta \gamma \tilde{\dot{x}}|\hat{z}|^{n}=c_{2}\left[(\Delta \gamma)^{2}\left(\tilde{\dot{x}}|\hat{z}|^{n}\right)^{2}+\varepsilon^{2}\right], \\
& \varepsilon \Delta \beta|\tilde{\dot{x}}||\hat{z}|^{n} \operatorname{sign}(\hat{z})=c_{1}\left[(\Delta \beta)^{2}\left(|\tilde{\dot{x}}||\hat{z}|^{n}\right)^{2}+\varepsilon^{2}\right]
\end{aligned}
$$

are satisfied in the area $O_{v}(O)$, where $O=\left\{0,0^{2}\right\} \subset R \times R^{2} \times J_{0, \infty}, \quad O_{v}$ is some neighbourhood of the point $O$; 4) inequality $\left(\varepsilon-\varepsilon_{z}\right)^{2 n} \geq c_{z}$ holds for almost all $t$ where $\left.c_{z} \geq 0 ; 5\right)$ such $\pi_{x} \geq 0$ and $\omega>0$ exist that $(\tilde{\dot{x}})^{2} \geq \pi_{x}$ and $\left.\left|\varepsilon-\varepsilon_{z}\right| \leq \omega|\varepsilon| ; 6\right)$ the function

$$
g_{2}(t)=\sup _{\varepsilon \in \Omega} \frac{|\varepsilon|^{2(n+1)}(t)}{V_{\varepsilon}(t, \varepsilon)}, \quad g_{2}=\sup _{\varepsilon \in \Omega} \tilde{g}_{2}(t)
$$

exists, where $\Omega$ the subsystem $A S_{Z}$ definition domain; 7) $\dot{V}_{\varepsilon}, \dot{V}_{\beta, \gamma}$ satisfy the system of inequalities

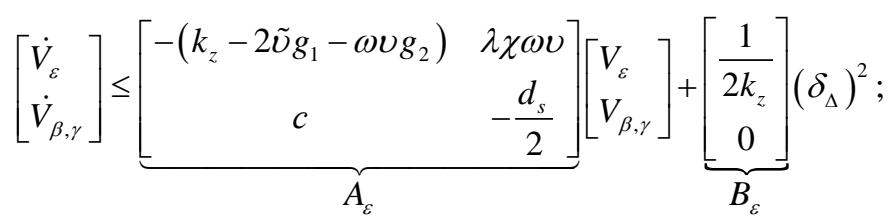

8) the upper solution for $V_{\varepsilon, \beta, \gamma}=\left[V_{\varepsilon}(t) V_{\beta, \gamma}(t)\right]^{\mathrm{T}}$ satisfies to the equation

$$
\dot{\tilde{S}}=A_{\varepsilon} \tilde{S}+B_{\varepsilon}\left(\delta_{\Delta}\right)^{2}
$$

if

$$
V_{\tilde{\rho}}(t) \leq \tilde{s}_{\tilde{\rho}}(t), \quad \forall\left(t \geq t_{0}\right) \&\left(V_{\tilde{\rho}}\left(t_{0}\right) \leq \tilde{s}_{\tilde{\rho}}\left(t_{0}\right)\right),
$$

where $\tilde{\rho}=\varepsilon, \beta, \gamma, \tilde{S}=\left[\begin{array}{cc}\tilde{s}_{\varepsilon} & \tilde{s}_{\beta, \gamma}\end{array}\right]^{\mathrm{T}}, A_{\varepsilon} \in R^{2 \times 2}$ is $M$-matrix. Then the system $A S_{Z}$ is exponentially dissipative with the estimation

$$
V_{\varepsilon, \beta, \gamma}(t) \leq \mathrm{e}^{A_{\varepsilon}\left(t-t_{0}\right)} \tilde{S}\left(t_{0}\right)+\left(\delta_{\Delta}\right)^{2} \int_{t_{0}}^{T} \mathrm{e}^{A_{\varepsilon}(t-\tau)} B_{\varepsilon} \mathrm{d} \tau,
$$

if $\left(k_{z}-2 \tilde{v} g_{1}-\omega v g_{2}\right) d_{s}>2 c \lambda \chi \omega v, k_{z}>2 \tilde{v} g_{1}-\omega v g_{2}, d_{s}>0$,

$$
\bar{\chi}=\min \left(\chi_{\beta}, \chi_{\gamma}\right), \bar{c}=\min \left(c_{1}, c_{2}\right), \chi=\max \left(\chi_{\beta}, \chi_{\gamma}\right), d_{s}=\chi \pi_{\chi} \bar{c} c_{z} .
$$

$M$-matrix is considered in [25].

So, the system $A S_{Z}$ is exponentially dissipative. The dissipativity area depends on the informational set $\mathrm{I}_{o}$ of the $S_{B W}$-system.

\subsection{Simulation Results}

Consider the system (1)-(3) with parameters $n=1.5, c=2, m=1, \beta=0.5$, $\alpha=0.7, k=0.6, d=a=1$. Parameters are selected based on simulation. The exciting force $f(t)=2-2 \sin (0.15 \pi t)$. The system is modeled with initial conditions $x(0)=1, \dot{x}(0)=0, z(0)=1$. Form the set $\mathrm{I}_{0}$. The system phase portrait and output of the hysteresis shown in Figure 1. 


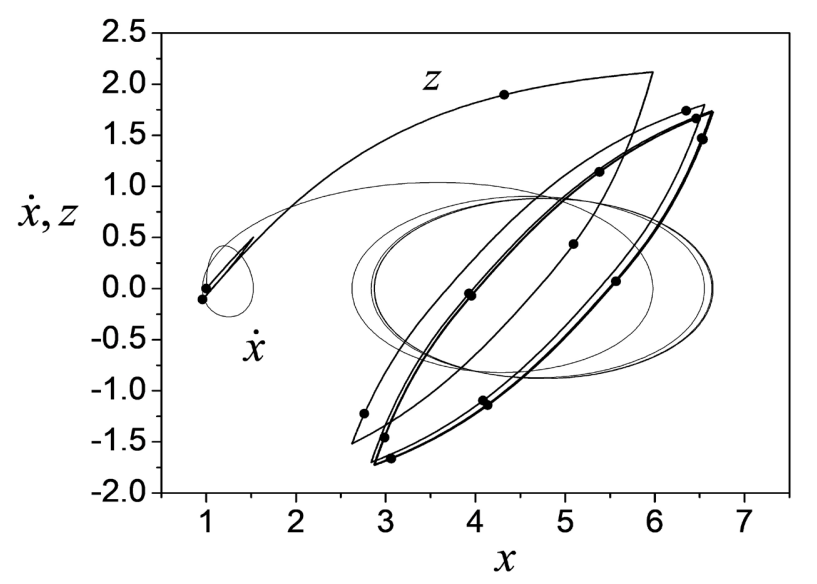

Figure 1. System phase portrait and hysteresis change.

Consider the identification of the system parameters. Determine the parameter $\mu$ of the system (13) using the transient process analysis for $\tilde{e}$ and $t<9.85 \mathrm{~s}$. Calculate Lyapunov exponents (LE) [26]. The estimation for the maximum LE is -0.9 . Therefore, we set $\mu=0.8$. Initial conditions in (7) are equal to zero.

Adaptive system work results presented in Figures 2-4. Parameters $k_{x}, k_{z}$ are equal to 2.5 and 0.75 . The tuning process of $A S_{X}$-systems (the model (8)) parameters shown in Figure 2. Figure 3 shown the model (11) parameters tuning.

Show the modification of identification errors $e, \varepsilon$ in Figure 4. We see that the accuracy of obtained estimations depends on the numbers of tuned parameters and the level $\dot{x}$ and properties $f(t)$. Obtained results confirm statements of theorems 3, 4. The $A S_{Z}$-system work results influence the tuning processes in the $A S_{X}$-system. Gain coefficients in (15), (16) and (17) are $\chi_{\beta}=0.0000002$, $\chi_{\gamma}=0.0000002, \gamma_{4}=0.00005 \gamma_{1}=0.0002, \gamma_{2}=0.00001, \gamma_{3}=0.00002$. The hysteresis output estimation shown in Figure 5.

So, simulation results confirm the exponential dissipativity of the designed system.

\section{Modification $S_{B W^{-} \text {-Systems }}$}

Various modifications of BWM have been proposed (see, for example, [4] [5] [26] [27] [28]). They reflect the features and properties of the control object. System (1)-(3) is the basis for modifications. The BWM modification proposes for the case of asymmetric hysteresis in [29]. The model has the form

$$
\dot{z}=\left(a-(\beta+\gamma \operatorname{sign}(z \dot{x}))|z|^{n}\right) \dot{x} .
$$

The BWM modifications set is based on the introduction of new multipliers in (3) [4] [30]. They reflect requirements to the system. BWM considering the degradation and clamping of reinforced concrete structures has the form [30]

$$
\dot{z}=\frac{h(z, \varepsilon)}{1+\delta_{\eta}}\left[\left(\dot{x}-1-\delta_{v}\right)\left(\beta|\dot{x}||z|^{n-1}+\gamma(\dot{x}|z|)^{n}\right)\right],
$$




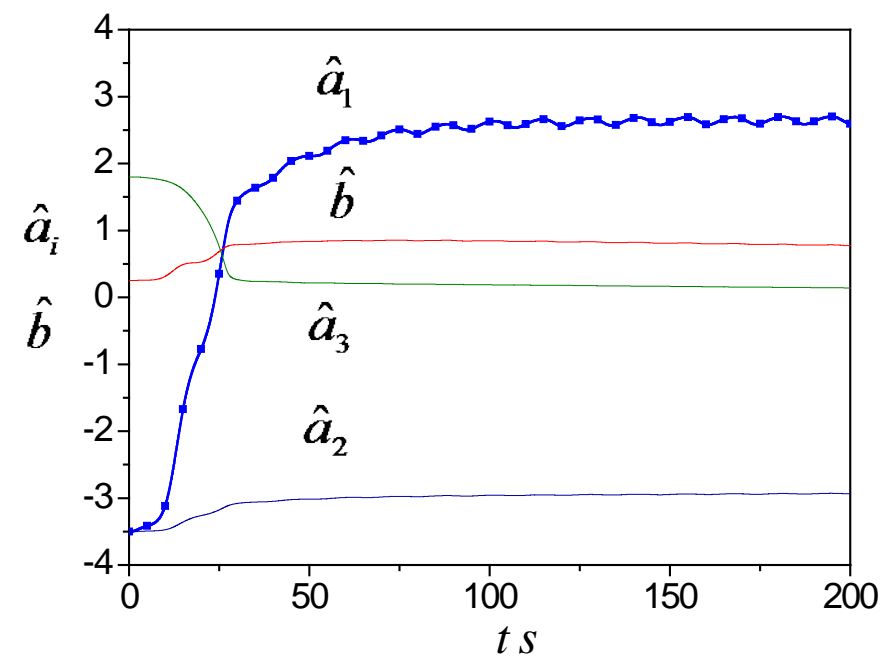

Figure 2. Tuning of model (8) parameters.

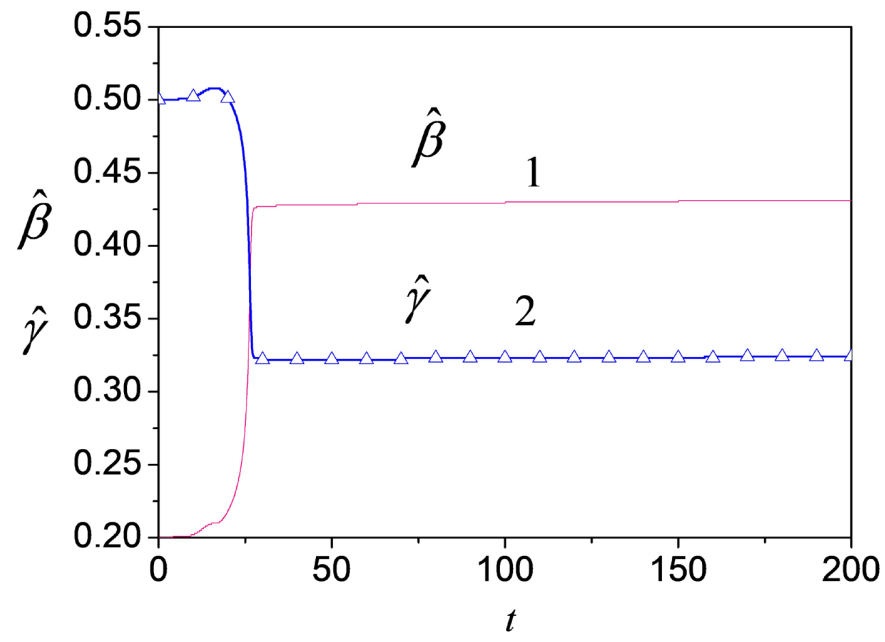

Figure 3. Tuning of model (11) parameters: 1 is tuning $\hat{\beta}, 2$ is tuning $\hat{\gamma}$.

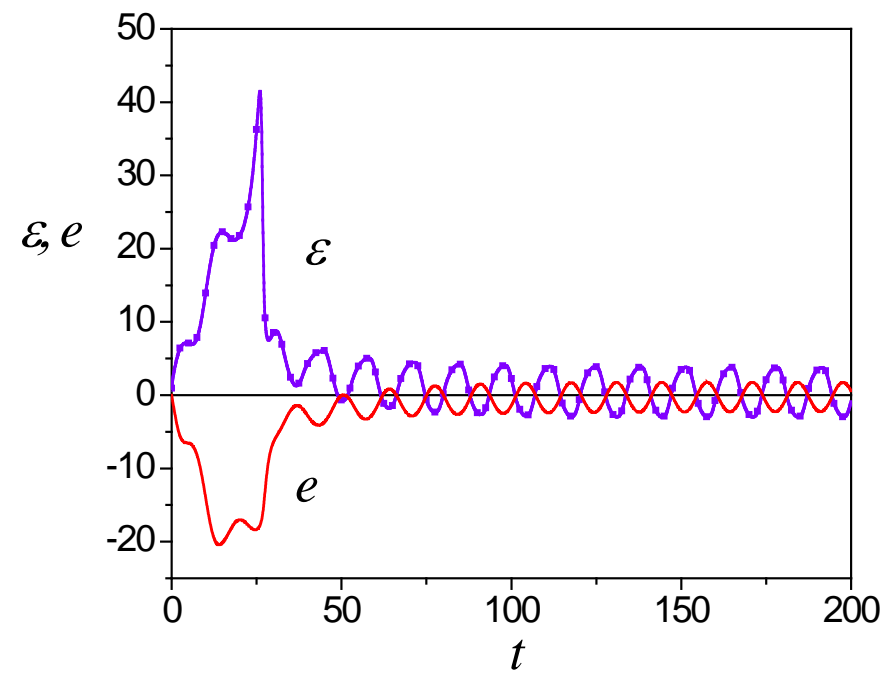

Figure 4. Outputs modification of systems $A S_{X}, A S_{Z}$. 


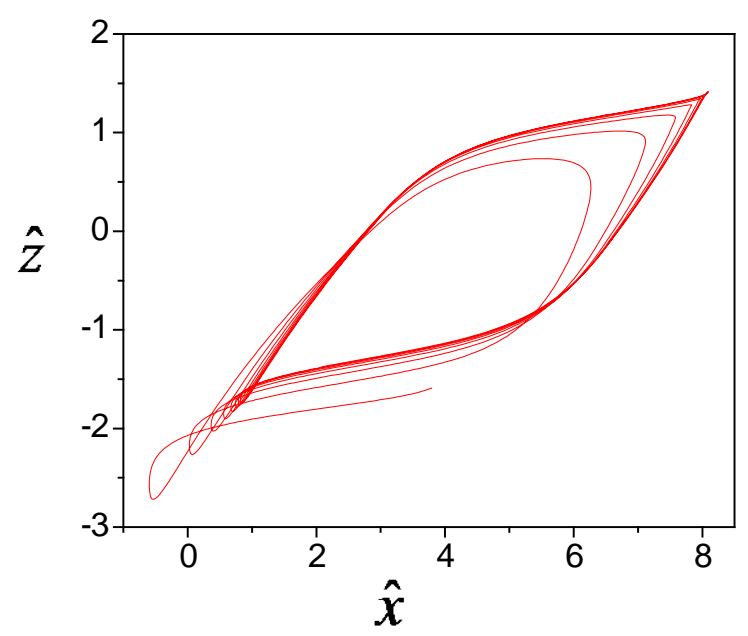

Figure 5. Hysteresis estimation at adaptation of $A S_{B W}$-system.

where $\delta_{\eta}$ and $\delta_{v}$ are parameters reflecting the decrease in rigidity and strength of the structure, $h(z, \varepsilon)$ considers the pinching effect.

The analysis showed that the last term in (3) is responsible for "fine-tuning" the hysteresis in the saturation or switching areas. If this is not critical for the object, then by selecting the parameters of the SW-system, this term in Equation (3) can compensate. In addition, some modifications simplify and increase the system (1)-(3) stability. They have the form [32]

$$
\begin{gathered}
\mathcal{M}_{\rho \omega \mu \nu \beta n}: \dot{z}=-\rho z|\dot{x}|^{\omega}+a|\dot{x}|^{\mu} \operatorname{sign}(\dot{x})-\beta|\dot{x}|^{\nu}|z|^{n} \operatorname{sign}(z), \\
\mathcal{M}_{\mu \beta n}: \dot{z}=a|\dot{x}|^{\mu} \operatorname{sign}(\dot{x})-\beta|\dot{x}||z|^{n} \operatorname{sign}(z), \\
\mathcal{M}_{\mu \nu \beta n}: \dot{z}=a|\dot{x}|^{\mu} \operatorname{sign}(\dot{x})-\beta|\dot{x}|^{\nu}|z|^{n} \operatorname{sign}(z) .
\end{gathered}
$$

The introduction in (42) of the linear component of $z$ increases the feasibility of the BWM and the $S_{B W}$-system stability. As the system is nonlinear, the function $|\dot{x}(t)|^{\omega}$ introduces to ensure the required hysteresis state. It guarantees a change $z$ in the specified boundaries. Parameters $\rho>0, \omega \geq 0$ are some numbers.

A comparison of the models (42)-(44) and BWM is shown in Figure 6. The representation allows comparing model properties by generalized indicators in the "minimum-maximum" space. Notation in Figure 6: $\mathrm{z}$ is model (3), $\mathrm{z} 1$ is model $\mathcal{M}_{\rho \omega \mu \nu \beta n}, \mathrm{z} 2$ is model $\mathcal{M}_{\mu \beta n}, \mathrm{z} 3$ is model $\mathcal{M}_{\mu \nu \beta n} ;$ is average value; - is median; $\bigcirc$ is the extreme value (end of the "saturation" region).

So, BWM modifications are considered. The application of proposed models depends on the object properties. The parameters influence analysis of models (42)-(44) give in [31].

\section{Theoretical Foundations of SI}

\subsection{Preliminary}

The modern direction of structural identification is based on the parametric 


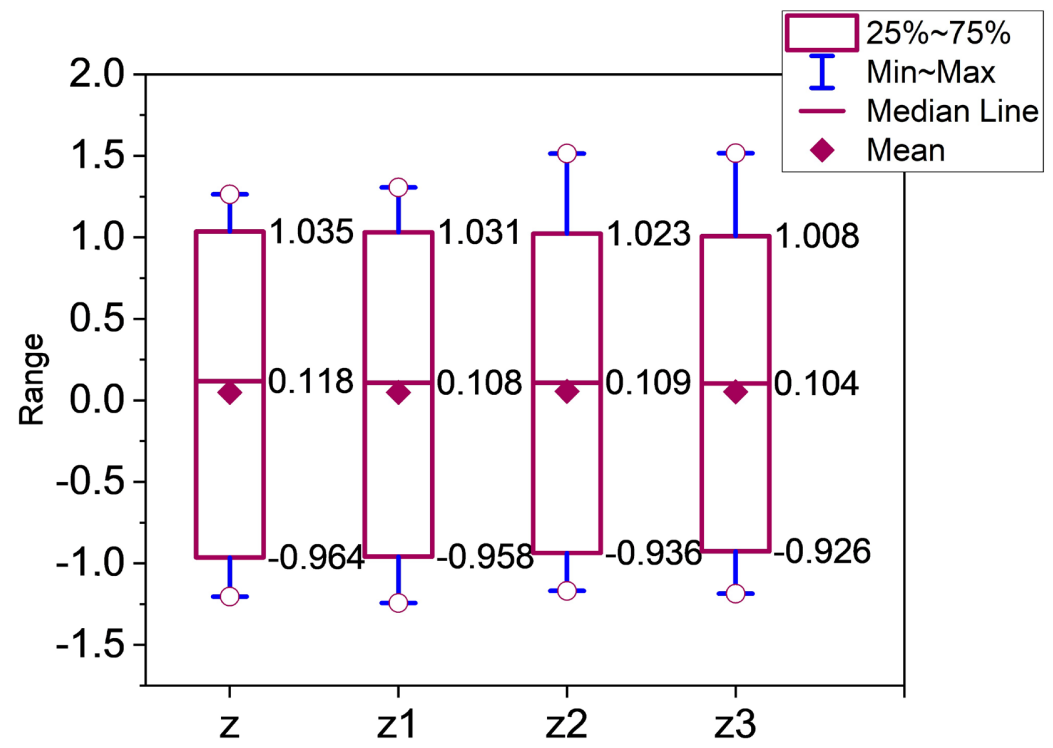

Figure 6. Comparison of hysteresis models (3), (42)-(44).

paradigm. It is explained by the formation and development of the theory of identification. Nonlinear systems SI methods are based on the approximation of nonlinearity by parametric models (see, for example, [32] [33] [34] [35] [36]). This approach leads to levelling of the nonlinearity structure. The second direction of structural identification is related to the analysis of geometric frameworks (GF). GF reflect the state of the system nonlinear part. It is the new direction in the identification theory. This approach proposes in [22] [37]. The statement of this approach gives below.

\subsection{Problem Statement}

Consider dynamic system

$$
\begin{aligned}
& \dot{X}=A X+\varphi(y) I+B u, \\
& y=C^{\mathrm{T}} X,
\end{aligned}
$$

where $u \in R, y \in R$ are input and output system; $A \in R^{q \times q}, B \in R^{q}, I \in R^{q}$ $C \in R^{q} ; \varphi(y)$ is the scalar nonlinear function belonging to the class of the hysteresis $F_{h} ; \quad I=[0,0, \cdots, 0,1]^{\mathrm{T}}$. We suppose that $A$ is the Hurwitz matrix.

Various assumptions are made about the structure of the function $\chi=\varphi(y)$. They determine by the level of a priori information. Under a priori definiteness, apply the methods based on linearization [38]. In the absolute stability study of nonlinear systems, suppose [28]

$$
\chi \in F_{\varphi}=\left\{\varphi(\xi) \xi \geq \xi^{2}, \xi \neq 0, \varphi(0)=0\right\},
$$

where $\xi \in R$ is the nonlinearity input. $\xi$ is a linear combination of the state variables (the vector $X$ ). The sector condition is used for approximation of function $\chi$

$$
\chi \in F_{\varphi}=\left\{\gamma_{1} \xi^{2} \leq \varphi(\xi) \xi \leq \gamma_{2} \xi^{2}, \xi \neq 0, \varphi(0)=0, \gamma_{1} \geq 0, \gamma_{2}<\infty\right\},
$$


Static nonlinearity often applies in control systems. Therefore, next, we consider the static (algebraic) functions which describe a hysteresis. For system (45), we have a set of the data

$$
\mathrm{I}_{o}=\left\{u(t), y(t), t \in J=\left[t_{0}, t_{k}\right]\right\} .
$$

Problem: determine a form and parameters of function $\varphi(y) \in F_{h}$ based of the analysis and a processing of the set $I_{o}$.

The problem solution is based on the formation of the set $\mathrm{I}_{N, g}$ contained data about $\varphi(y)$.

\subsection{Formation of Set $\mathbf{I}_{N, g}$}

The differentiation operation applies to $y(t)$ and designates the obtained variable as $x_{1}$. Generate informational the set $\mathrm{I}_{e n t}=\left\{\mathrm{I}_{o}, x_{1}\right\}$. Select the data $\mathrm{I}_{g} \subset \mathrm{I}_{e n t}$ subset described the particular solution (steady state) of the system (45). The mathematical model

$$
\hat{x}_{1}^{l}(t)=H^{\mathrm{T}}[1 u(t) y(t)]^{\mathrm{T}}
$$

applies to obtain $I_{g}=I_{e n t} \backslash I_{t r}$. Model (49) is determined on the time gap $J_{g}=J \backslash J_{t r}$ and gives the linear component $x_{1}$ estimation. $H \in R^{3}$ is a parameters model vector. The choice of an interval $J_{g}$ depends on the value of criterion $Q(e)$.

Determine a vector $H$ as

$$
\left.\min _{H} Q(e)\right|_{e=\hat{x}_{1}^{l}-x_{1}} \rightarrow H_{o p t},
$$

where $Q(e)=0.5 e^{2}$.

Apply the model (49) and determine the forecast for the variable $x_{1}(t)$ $\forall t \in \mathrm{I}_{g}$. Compute the error $e(t)=\hat{x}_{1}^{l}(t)-x_{1}(t) \cdot e(t)$ depends on nonlinearity $\varphi(y)$ in the system (45). Obtain set

$$
\mathrm{I}_{N, g}=\left\{y(t), e(t), t \in J_{g}\right\},
$$

which we will use next. We will apply the designation $y(t)$, supposing that $y(t) \in \mathrm{I}_{N, g}$.

The further problem solution is based on the analysis of frameworks $S_{e y}$, $S_{e k}$ which reflect the state of the nonlinearity.

Remark 4. Choice of the model (49) structure is one of the stages of structural identification. Simulation results show that the model (49) is used in identification systems of plants with static nonlinearity. For other classes of nonlinearity, this problem demands further research.

\subsection{Frameworks $S_{e y}, S_{e k}$}

Go into space $\mathscr{P}_{y e}=(y, e)$ and construct the phase portrait $S$ of the system (45). The framework $S_{e y}$ corresponds to a phase portrait $S$ [37]. $S_{e y}$ describes function $\Gamma_{e y}:\{y\} \rightarrow\{e\} \quad \forall t \in J_{g} . S_{e y}$ must have a closed form. This 
property $S_{e y}$ differs from frameworks $S_{e k} \cdot S_{e k}$ is applied for the analysis of statics systems [39] [40].

For decision making, we will use also $S_{e k}$-framework. $S_{e k}$ is described by function $\Gamma_{e k}:\left\{k_{s}(t)\right\} \rightarrow\{e(t)\}$, where $k_{s}(t) \in R$ is a coefficient of structural properties [39] systems (45) in space $\mathscr{P}_{y e}$

$$
k_{s}(t)=\frac{e(t)}{y(t)} .
$$

Next, we construct sector sets for system (45) in the space $\mathscr{P}_{k e}=(k, e)$ and will be decision-making on the class $F_{h}$. The solution to this problem is based on the analysis of proposed frameworks.

\subsection{About Properties $\mathbf{I}_{N, g}$}

Consider the set $\mathrm{I}_{N, g}$ properties ensured the solution of hysteresis F1 structural identification. Let fulfill to conditions

(i) the set $I_{o}$ ensures the solution of the model (49) parametric identification problem.

(ii) the input $u(t)$ ensures obtaining informative framework $S_{e y}\left(\mathrm{I}_{N, g}\right)$ or $S_{e k}\left(\mathrm{I}_{N, g}\right)$.

If $u(t)$ has properties (i), (ii), then input $u(t)$ is representative.

Let the framework $S_{e y}$ is closed and its area is not zero. Designate altitude $S_{e y}$ as $h\left(S_{e y}\right)$, where the altitude is the distance between two points of the opposite sides of framework $S_{e y}$. Then the framework $S_{e y}$ is identified on set $\mathrm{I}_{N, g}$.

Let $u \in \mathscr{P} E_{\alpha}$, where $\mathscr{P} E_{\alpha}$ is the constancy excitation property

$$
P E_{\alpha}: u^{2}(t) \geq \alpha
$$

fair for $\exists \alpha>0$ and $\forall t \geq t_{0}$ on some interval $T>0$.

Statement 1 [37]. Let (i) the linear part of system (45) is stable and nonlinearity satisfies the condition (47); (ii) the input $u(t)$ is piecewise continuous, limited and constantly exciting; (iii) exists $\delta_{S}>0$ such that $h\left(S_{e y}\right) \geq \delta_{S}$. Then the framework $S_{e y}$ is identified on set $\mathrm{I}_{N, g}$.

Proof of Statement 1. Consider input $u(t)$ satisfied to condition 1). $u(t)$ corresponds Fourier series containing a sinusoid with frequency $\omega_{i}$. The output $y(t) \in \mathrm{I}_{N, g}$ contains components of this spectrum and has a phase shift. The variable $x_{1}$ is the result of the differentiation $y(t)$. Hence, $x_{1}$ contains components with this frequent spectrum. Therefore, the framework $S$ (phase portrait) on a phase plane $\left(y, x_{1}\right)$ has a closed form. The $S_{e y}$-framework has the same form. Determine the distance $h\left(S_{e y}\right)$ between opposite points of the framework $S_{e y} \cdot y(t), x_{1}(t)$ satisfy the condition 2) statement 1 . Therefore, for almost all $t \in J_{N, g} h\left(S_{e y}\right)>\delta_{S}$.

The framework $S_{e y}$ which has referred properties, we will name $h$-identified. Further, we believe that $S_{e y}$ has the specified properties.

Features of the $h$-identifiability. 
1) $h$-identifiability is a concept not parametric identification, and structural identification;

2) The demand for parametric identifiability is the base $h$-identifiability;

3) $h$-identifiability determines more stringent demands to the system input.

Feature 3 means that "the bad" input can satisfy a constancy excitation condition. Such input can give a so-called "insignificant" $S_{e y}$-framework (framework $\mathcal{N} S_{\text {ey }}$ ) [37] which will have property $h$-identifiability.

\subsection{Framework $\mathcal{N} S_{e y}$}

Consider the framework $S_{e y}$. Let $S_{e y}=F_{S_{e y}}^{l} \cup F_{S_{e y}}^{r}$, where $F_{S_{e y}}^{l} F_{S_{e y}}^{r}$ are left and right fragments $S_{e y}$. Determine for $F_{S_{e y}}^{l}, F_{S_{e y}}^{r}$ secants

$$
\gamma_{S}^{l}=a^{l} y, \quad \gamma_{S}^{r}=a^{r} y,
$$

where $a^{l}, a^{r}$ are the numbers determined using the method of least squares (LSM).

Theorem 5 [37]. Let (i) the framework $S_{\text {ey }}$ is $h$-identifiable; (ii) the framework $S_{e y}$ have the form $S_{e y}=F_{S_{e y}}^{l} \cup F_{S_{e y}}^{r}$, where $F_{S_{e y}}^{l}, F_{S_{e y}}^{r}$ are left and right fragments $S_{e y}$; (iii) for $F_{S_{e y}}^{l}, F_{S_{e y}}^{r}$ secants (54) are obtained. Then $S_{e y}$ is $\mathcal{N} S_{e y}$ framework, if

$$
\left|a^{l}-a^{r}\right|>\delta_{h}
$$

where $\delta_{h}>0$ is some number.

Theorem 5 proves based on sets homothety.

Remark 5. $\quad \mathcal{N S}_{e y}$-frameworks are characteristic for systems with many-valued nonlinearities. They are the input inadequate use result.

\section{Structural Identification and Structural Identifiability BWH}

We have noted (see introduction) that structural identifiability (SID) is the result of structural identification. Therefore, we will consider the SID basics guaranteed SI.

Apply the SI model (49) and represent the system (45) as (system $S_{y \varphi}$ )

$$
\left\{\begin{array}{l}
S_{y}:\left\{\begin{array}{l}
\dot{\tilde{X}}=A \tilde{X}+I \zeta, \\
\tilde{y}=C^{\mathrm{T}} \tilde{X},
\end{array}\right. \\
S_{\varphi}: e=f\left(y, x_{1}\right),
\end{array}\right.
$$

where $\tilde{X} \in R^{n}$ is a variable describing the general solution of the system (45), $\zeta \in R$ is a bounded perturbation appearing as the analysis result of the variable $e$.

\subsection{System $S_{\varphi}$}

Consider the identifiability problem system $S_{\varphi}$. Let conditions hold.

$B 1$. The input is constantly excited at the interval $J$. 
B2. The analysis of $S_{e y}$ gives the solution to the estimation problem the nonlinear properties of the system $S_{y \varphi}$.

We state the basic concepts, generalizing the results [22].

Definition 2. If $u(t)$ satisfies B1 and B2 conditions, then the input $u(t)$ is representative.

Let the framework $S_{e y}$ closed, and the area $S_{e y}$ is not zero. Denote height $S_{e y}$ as $h\left(S_{e y}\right)$ where height is the distance between two points opposite sides of the framework $S_{e y}$.

Theorem 6 [37]. Let (i) the linear part of the system (45) is stable; (ii) the nonlinearity $\varphi(\cdot)$ satisfies the condition (47); (iii) the input is bounded and constantly excited; 4) $h\left(S_{\text {ey }}\right) \geq \delta_{S}$, where $\delta_{S}>0$. Then the framework $S_{\text {ey }}$ is identified on the set $\mathrm{I}_{N, g}$.

Definition 3. The framework $S_{e y}$ is called $h$-identifiable if theorem 6 holds for $S_{e y}$.

Let $S_{e y}$ be $h$-identifiable. Introduce designations: $\mathscr{D}_{y}=\operatorname{dom}\left(S_{e y}\right)$ is definition range of the framework $S_{e y}, D_{y}=D_{y}\left(D_{y}\right)=\max y(t)-\min y(t)$ is diameter $\mathcal{D}_{y}$. Let $u(t) \in \mathrm{U}$, where $\mathrm{U}$ is an acceptable set of inputs for the system (45). The set $U$ contains representative inputs.

Definition 4. If $\mathcal{D}_{y}$ of the framework $S_{e y}$ has a maximum diameter $D_{y}$, the input S-synchronizes the system (45).

Consider a reference framework $S_{e y}^{r e f} \cdot S_{e y}^{r e f}$ is the framework $S_{e y}$ reflecting all properties of the function $\varphi(y)$. Designate by the diameter $D_{y}\left(S_{e y}^{r e f}\right)$ as $D_{y}^{r e f} . D_{y}^{r e f}$ exists if the input the system (45) is S-synchronizing.

Definitions 2,3 show if $S_{e y} \cong S_{e y}^{r e f}$, then $\left|D_{y}-D_{y}^{r e f}\right| \leq \varepsilon_{y}$, where $\varepsilon_{y} \geq 0$, is the proximity sign. Elements of the subset $U_{S}$ have property

$$
\left|D_{y}\left(S_{e y}\left(\left.u(t)\right|_{u \in \mathrm{U}_{\mathrm{S}}}\right)\right)-D_{y}^{r e f}\right| \leq \varepsilon_{y} .
$$

Synchronization $u(t) \in \mathrm{U}$ is the choice of such input $u_{h}(t) \in \mathrm{U}$ that reflects all features $\varphi(y)$ in $S_{e y}$. It is true if $u(t)$ ensures $\max _{u_{h}} D_{y}$ and $S_{\text {ey }} \neq \mathcal{N} S_{\text {ey }}$. We interpret the choice $u_{h}(t) \in \mathrm{U}$ as ensuring synchronization between structures of a model and the system. $d_{h, y}=\max _{u_{h}} D_{y}$ is the condition of $h$-identifiability which can represent as

$$
\left|D_{y}\left(S_{e y}\left(\left.u(t)\right|_{u \in \mathrm{U}_{\mathrm{S}}}\right)\right)-d_{h, y}\right| \leq \varepsilon_{y} .
$$

The condition for $\mathcal{N} S_{e y}$

$$
\left|D_{y}\left(S_{e y}\left(\left.u(t)\right|_{u \in \mathrm{UIU}_{\mathrm{S}}}\right)\right)-d_{h, y}\right|>\varepsilon_{y} .
$$

(58) can be interpreted as proximity domain

$$
Q_{D}=\left|S_{e y}\left(\left.u(t)\right|_{u \in \mathrm{U}_{\mathrm{S}}}\right)-S_{e y}^{r e f}\right|,
$$

which is understood as $\left|\dot{y}(t)-\dot{y}^{\text {ref }}(t)\right| \leq \varepsilon_{y}$ for almost $\forall t \geq \tilde{t}$.

We will write $\delta Q_{D} \leq \varepsilon_{y}$ if considered frameworks are close.

Domain $Q_{D}$ is the S-synchronizability area on $\left\{u_{h}(t)\right\}$ or the structural 
identifiability domain on $\left\{S_{h}\left(u_{h}(t)\right)\right\}$, where $S_{h}$ is the phase portrait of the system (45) if the condition $\delta Q_{D} \leq \varepsilon_{y}$ is true for $Q_{D}$ almost $\forall t \geq t^{*}$.

So, two criteria (55) and (59) presented for the existence of the insignificant framework $\mathcal{N} S_{e y}$. Structure of systems $S_{\varphi}$ and (45) are structurally non-identifiable in this case.

Let the input $u_{h}(t)$ synchronize the system (45). If $u(t)$ is S-synchronizing, then we will write $u_{h}(t) \in \mathrm{S}$. Note that a finite set $\left\{u_{h}(t)\right\} \in \mathrm{S}$ exists for the system (45). The choice of optimal $u_{h}(t)$ depends on $d_{h, y}$ and (58). The hold of the condition (58) is one of the prerequisites for SI of the system (45).

Definition 5. If framework $S_{e y}$ is $h$-identified and conditions $\left\|a^{r}|-| a^{r}\right\| \leq \delta_{h}$, (8) are satisfied, then the framework $S_{e y}$ or the system (56) (system (45)) is structurally identified or $h_{\delta_{h}}$-identifiable.

Remark 6. Conditions specified in definition 5 are the conditions for the structural identification of systems (45), (56).

Definition 5 shows if the system (45) is $h_{\delta_{h}}$-identified then the framework $S_{e y}$ has the maximum diameter of area $\mathcal{D}_{y}$.

Definition 6. The model (49) is $S M$-identifying if the framework $S_{e y}$ is $h_{\delta_{h}}{ }^{-}$ identifiable.

The framework $S_{e y}$ is defined on $u_{h}(t) \in \mathrm{S}$ and $u_{h}(t)$ satisfies condition B1. Therefore, $S_{e y}$ corresponds to the nonlinearity $\varphi(y)$ defined on the class

$$
\varphi(y) \in F_{\varphi}=\left\{\varphi(y) \in R \mid \varphi(y, \mathrm{~A}), \mathrm{A} \in R^{n_{\AA}}, \alpha_{i} \in \mathrm{A}, \alpha_{i} \in\left[\bar{\alpha}_{i}, \overline{\bar{\alpha}}_{i}\right]\right\},
$$

where $\bar{\alpha}_{i}, \overline{\bar{\alpha}}_{i}$ are some numbers.

Note that the term $S M$-identifying does not coincide with the concept proposed in [41].

Theorem 7 [42]. Let (i) the input $u(t) \in \mathrm{S}$ is constantly excited; (ii) the system (45) phase portrait have $m$ features; (iii) $S_{e y}$-framework is $h_{\delta_{h}}$-identified and contains fragments corresponding to features of the system (45). Then the model (49) is $S M$-identifying.

The theorem 7 shows if the model (49) is not $S M$-identifying then model (49) structure or the informational set (48) need to change.

Let $c_{S}$ is the center of the framework $S_{e y}$ on the set $J_{y}=\{y(t)\}, \quad c_{D_{y}}$ is the center of the area $\mathcal{D}_{y}$.

Theorem 8. Let the set $\mathrm{U}_{\mathrm{S}}$ given for the system $S_{y \varphi}$ and (i) exists $\varepsilon \geq 0$ such that $\left|c_{s}-c_{D_{y}}\right| \leq \varepsilon$; (ii) $\left\|a^{l}|-| a^{r}\right\| \leq \delta_{h}$, where $a^{l}, a^{r}$ are coefficients of secants (54) for $\left(F_{S_{e y}}^{l}, F_{S_{e y}}^{r}\right) \subset S_{e y}$. Then the system (56) is $h_{\delta_{h}}$-identifiable, the input $u_{h}(t) \in \mathrm{S}$, and the framework $S_{e y}$ defines the class $F_{\varphi}$.

Proof of Theorem 8. Consider the input $u_{h}(t) \in \mathrm{U}_{\mathrm{S}}$. Since condition $\left|a^{l}-a^{r}\right| \leq \delta_{h}$ is satisfied, the framework $S_{e y}$ is symmetric concerning the point $c_{s}$ plane $(y, e)$. Consequently, definitional domains diameters of the fragments $F_{S_{e y}}^{l}, F_{S_{e y}}^{r}$ for the framework $S_{e y}$ coincide up to a certain value $\varepsilon_{F} \geq 0$ on the set $\{y(t)\}$, i.e. 


$$
\left|D_{F_{S}^{l}}\left(\mathcal{D}_{F_{S}^{l}}\right)-D_{F_{S}^{r}}\left(\mathcal{D}_{F_{S}^{r}}\right)\right| \leq \varepsilon_{F}
$$

where $\mathcal{D}_{F_{S}^{l}}, \mathcal{D}_{F_{S}^{r}}$ are definitional domains $F_{S_{e y}}^{l}, F_{S_{e y}}^{r}$. Then the framework $S_{e y}$ centre is equal to $c_{D_{y}}=0.5\left(D_{F_{S}^{l}}+D_{F_{S}^{r}}\right)$. Since $D_{F_{S}^{l}}+D_{F_{S}^{r}}=D_{y}$, there exist $\varepsilon \geq 0$ such that $\left|c_{S}-c_{D_{y}}\right| \leq \varepsilon$. The fulfillment of conditions (i), (ii) guarantees $u(t)=u_{h}(t)$ and $d_{h, y}=\max _{u_{h}} D_{y}$. Therefore, the framework $S_{e y}$ contains all the features characteristic of the function $\varphi(y)$ at $u_{h}(t)$. So, $u_{h}(t) \in \mathrm{S}$, and system $(45)$ is $h_{\delta_{h}}$-identifiable.

As $\varphi(y) \in F_{\varphi}$, then the area $\mathcal{D}_{y}$ have center $c_{D_{y}} \in J_{c_{D_{y}}}, J_{c_{D_{y}}}$ is some interval.

Len subset $\left\{u_{h, i}(t)\right\} \subset \mathrm{U}_{\mathrm{S}} \subseteq \mathrm{U} \quad(i \geq 1)$ which elements have the property of S-synchronizability exists. The framework $S_{e y, i}\left(u_{h, i}\right)$ has the diameter $D_{y, i}\left(\mathcal{D}_{y, i}\right)$ and corresponds to every $u_{h, i}(t)$. As $u_{h, i}(t) \in \mathrm{S}$ the diameter $D_{y, i}$ has the property $d_{h, \Sigma}$-optimality.

Let the hypothetical framework $S_{e y}$ (a framework $S_{e y}^{r e f}$ ) of the system (45) have diameter $d_{h, \Sigma}$.

Definition 7. The framework $S_{e y, i}$ has $d_{h, \Sigma}$-optimality property on the set $\mathrm{U}_{h}$ if $\varepsilon_{\Sigma}>0$ such that $\left|d_{h, \Sigma}-D_{y, i}\right| \leq \varepsilon_{\Sigma} \quad \forall i=\overline{1, \# \mathrm{U}_{h}}$.

Definition 8. If $\left(\left\{u_{h, i}(t)\right\}=\mathrm{U}_{h} \subset \mathrm{U}\right) \&\left(u_{h, i}(t) \in \mathrm{S}\right), i \geq 1$ and frameworks $S_{e y, i}\left(u_{h, i}\right)$ have $d_{h, \Sigma}$-optimality property, then frameworks $S_{e y, i}\left(u_{h, i}\right)$ are structurally indiscernible on sets $\left\{u_{h, i}(t)\right\}$.

So, the $h_{\delta_{h}}$-identifiability estimate can be obtained from any input, following definitions 6,7 .

Definition 9. If frameworks $S_{e y, i}\left(u_{h, i}\right)$ have $d_{h, \Sigma}$-optimality property, then $S_{e y, i}\left(u_{h, i}\right)$ is locally structurally identifiable on the set $\mathrm{U}_{h}$.

Let the framework $S_{e y, i}\left(u_{h, i}\right)$ having $d_{h, \Sigma}$-optimality property is $S_{e y, i}^{\Sigma}$, and the locally structurally identified framework $S_{e y, i}\left(u_{h, i}\right)$ is $S_{e y, i}^{L S I}$.

The framework $S_{e y}$ is locally structurally identifiable on the set $\mathrm{U}_{h} \subseteq \mathrm{U}_{\mathrm{S}}$ if

$$
\left(\exists u_{h} \in \mathrm{S}\right) \text { what }\left(S_{e y} \cong S_{e y}^{\Sigma}\right) \rightarrow S_{e y} \cong S_{e y}^{L S I} .
$$

Remark 7. We consider nonlinearities satisfying condition (47). Therefore, notes made above are valid.

Definition 10. The framework $S_{e y, i}\left(u_{i} \notin \mathrm{U}_{\mathrm{S}}\right)$ that does not have the $d_{h, \Sigma}-$ optimality property is locally structurally non-identifiable on the set $\mathrm{U}_{h}$.

The framework $S_{e y, i}\left(u_{i} \notin \mathrm{U}_{\mathrm{S}}\right)$ that is structurally non-identifiable on the set $\mathrm{U}_{h}$ defines a class $F_{\varphi}^{N} \not \subset F_{\varphi}$.

Remark 8. The described approach applies to the nonlinear system with a dynamic law of nonlinearity change. In this case, the hierarchical immersion method [43] is used for the structure estimation of the nonlinearity.

The identifiability of system $S_{y}$ considered in [44]. Let the phase portrait $S$ constructed for the system. 


\subsection{Non-Identifiability Degree}

Obtain the non-identifiability degree estimate of the system (56). Definitional domains of $S$ and $S_{e y}$ are coincident. Therefore, the diameter $D\left(S_{e y}\right)$ of the framework $S_{e y}$ is known. Consider the set $\left\{u_{i}(t)\right\}$ having the property $\mathcal{P E}_{\alpha}$. Determine the framework $S_{e y, i}$ for each $u_{i}(t)$ and obtain $D_{y, i}\left(S_{e y, i}\right)$. Suppose $d_{h, y}=\max _{u_{i}}\left|D_{y}\left(\mathcal{D}\left(S_{e y, i}\right)\right)\right|$ and denote the corresponding input as $u_{h}$. Determine diameters $d_{y, j}=\left|D_{y, j}\left(\mathscr{D}\left[S_{e y, j}\left(u_{j} \in \mathcal{U}\right)\right]\right)\right|$ for all inputs $V=\left\{u_{i}(t)\right\} \backslash\left\{u_{h}\right\}$. Since $u_{h} \in \mathrm{S}$, therefore $\widehat{d}_{h, y}>D_{y, j} \quad \forall j \geq 1$. As $u_{h} \in \mathrm{S}$, therefore $d_{h, y}>D_{y, j} \quad \forall j \geq 1$. Then evaluate the non-identifiability degree as

$$
S I_{j}=S I\left(S_{e y, j}\right)=\frac{d_{h, y}-d_{y, j}}{d_{h, y}}
$$

$S I$ shows that $S_{B W}$-system (1) is structurally identifiable if $S I_{j} \rightarrow 0$.

If estimates for the fragments $\mathcal{F}_{S}^{l}, F_{S}^{r}$ of the phase portrait $S$ are known, then the identifiability degree is defined as

$$
S I=S I(S)=\frac{d_{y}^{l}\left(F_{s}^{l}\right)}{d_{y}^{r}\left(F_{s}^{r}\right)},
$$

where $d_{y}^{l}\left(F_{S}^{l}\right), d_{y}^{r}\left(F_{S}^{r}\right)$ are diameters of fragments $F_{S}^{l}, F_{S}^{r}$. The system $S_{\varphi}$ is structurally identifiable if $S I(S) \leq O(1)$ where $O(1)$ is neighborhood 1 .

Example 1. Consider the BWH from Section 3.4. Consider four variant inputs

$$
\begin{aligned}
& u_{0}(t)=2-2 \sin (0.15 \pi t), u_{1}(t)=2-2 \sin (0.35 \pi t), \\
& u_{2}(t)=2-2 \sin (0.5 \pi t), u_{3}(t)=2-2 \sin (0.15 \pi t)+0.2 \sin (0.35 \pi t) .
\end{aligned}
$$

Calculate diameters for the phase portrait definitional domain

$$
D_{y, 0}\left(S_{0}\right)=3.75, D_{y, 1}\left(S_{1}\right)=1.728, D_{y, 2}\left(S_{2}\right)=1.08, D_{y, 3}\left(S_{3}\right)=3.967 \text {. (67) }
$$

Results obtained for the system $S_{B W}$ steady state. The analysis showed $u_{0}(t) \in \mathrm{S}$. We assume that the system $S_{B W}$ with the phase portrait $S_{0}$ is the standard and $d_{h, y}=D_{y, 0}\left(S_{0}\right)$. The degree of non-identifiability of the system $S_{B W}$ for various $u_{i}$

$$
S I_{1}=0.549, S I_{2}=0.718, S I_{3}=-0.035 .
$$

We see that the $S_{B W}$-system is structurally non-identifiable with $u_{1}, u_{2}$, and the $S_{B W}$-system with input $u_{3}$ is structurally indistinguishable from input $u_{0}$. So, frameworks $S_{e y, 1}\left(u_{1}\right), S_{e y, 2}\left(u_{2}\right)$ are frameworks of class $\mathcal{N} S_{e y}$, and the framework $S_{e y, 3}\left(u_{3}\right)$ belongs to class $S_{e y}^{L S I}$.

\subsection{Hierarchical Immersion Method}

If nonlinear processes are complex, then the model (49) will be inadequate. Then the hierarchical immersion method (HIM) [44] is used to design the $S_{e y}$ framework. HIM realizes the subsequent stages of synthesis $S_{e y}$ if the model (47) is inadequate. The method is based on the application (49) in a new structural space and the synthesis for $\tilde{S}_{e y}$ new framework. If the new model (49) is significant, HIM stops. Otherwise, a new iteration is implemented. 


\subsection{SI and SID Bouc-Wen Hysteresis}

Consider the BWH from section 3.4. Introduce the framework $S_{\text {ey }}$ to estimate the $S_{B W}$-system structural identifiability. $S_{e y}$ is the hysteresis estimation in the structural space $\mathscr{P}_{e y}$. Apply the model

$$
\dot{\hat{x}}=-0.199 x+0.471 f
$$

and calculate the error $e=\dot{x}-\dot{\hat{x}}$.

The framework $S_{e y}$ described by the mapping $s_{e y}: y(t) \rightarrow e(t)$ and is showed in Figure 7.

Apply the approach proposed in [45]. Draw the straight line parallel to the ordinate axis through point $c_{S}$. Obtain two fragments $\left(F_{S_{e y}}^{l}, F_{S_{e y}}^{r}\right) \subset S_{e y}$. Determine secants for the left $F_{S_{e y}}^{l}$ and right $F_{S_{e y}}^{r}$ fragment

$$
\begin{aligned}
& \gamma_{e}^{l}=0.0313 y-0.146, r_{y e, l}^{2}=0.912, \\
& \gamma_{e}^{r}=0.032 y-0,15, r_{y e, r}^{2}=0.926
\end{aligned}
$$

Let $h\left(S_{e y}\right)$ be the distance between the opposite sides of the framework $S_{e y}$. The framework $S_{\text {ey }}$ satisfies conditions of theorem 6. The height $h\left(S_{e y}\right) \geq 0.02$, and the input $f(t)$ is constantly excited and S-synchronized. Therefore, the $S_{\text {ey }}$-framework (system $S_{B W}$ ) is $h_{\delta_{h}}$-identifiable. Figure 8 confirms this conclusion. Models (69) is $S M$-identifying.

Consider the structural identification of BWH. Apply the hierarchical immersion method for estimating the BWH structure. Calculate the derivative for $e$ applying numerical derivation. This procedure is sensitive to calculation errors. Therefore, perform smoothing $\dot{e}(t)$ applying the Fourier transform.

Denote the obtained variable as $\varepsilon=\dot{e}$. Further analysis has shown that $\varepsilon$ did not depend on $x$ (see Figure 8). Thus, $\varepsilon$ depends on $\dot{x}$ or $z$.

Consider the framework $S_{\varepsilon \tilde{x}}$ described by the mapping $\Gamma_{\varepsilon \hat{x}}: \hat{\dot{x}} \rightarrow \varepsilon$, where $\hat{\dot{x}}$ is the estimate of the derivative $\dot{x}$. Determine the secant $\gamma_{\varepsilon}$ for $S_{\varepsilon \tilde{x}}$ :

$$
\gamma_{\varepsilon \hat{\dot{x}}}=0.0324 \hat{\dot{x}}, r_{\varepsilon \hat{x}}^{2}=0.86 \text {. }
$$

The model (71) presents in Figure 8. Therefore, Figure 8 and the model (71) confirm effect $\dot{x}$ on hysteresis properties.

Estimate the relationship between variables $z$ and $\varepsilon$. Use the variable $e$ as the estimation $z$. Apply the denote $\hat{z}=e$. The analysis shows $\hat{z}$ and $\varepsilon$ not relates by the linear dependence. Therefore, the correlation between $\varepsilon$ and the combination $\hat{z}$ and $\hat{\dot{X}}$ exists. Eliminate the effect of the linear component $\hat{\dot{X}}$ on $\varepsilon$. Obtain the variable $\vartheta=\varepsilon-\gamma_{\varepsilon \hat{x}}$. Go to into the space $\mathscr{P}_{\vartheta_{\mu}}=(\vartheta, \mu)$, $\mu=|\hat{z}|^{h} \hat{\dot{x}}, h>0$.

The example of the relation estimation is shown in Figure 9, where $h=0.5$. The secant $\gamma_{\vartheta_{\mu}}$ framework $S_{\vartheta_{\mu}}$ has the form $\gamma_{\vartheta_{\mu}}=0.354 \mu$, coefficient of determination $r_{\vartheta_{\mu}}^{2}=0.82$. The parameter $h$ cannot correspond to the parameter $n$ in (3). The cause of such discrepancy follows from the proposed approach. True of BWM parameters estimates based on the use of the parametric identification. 


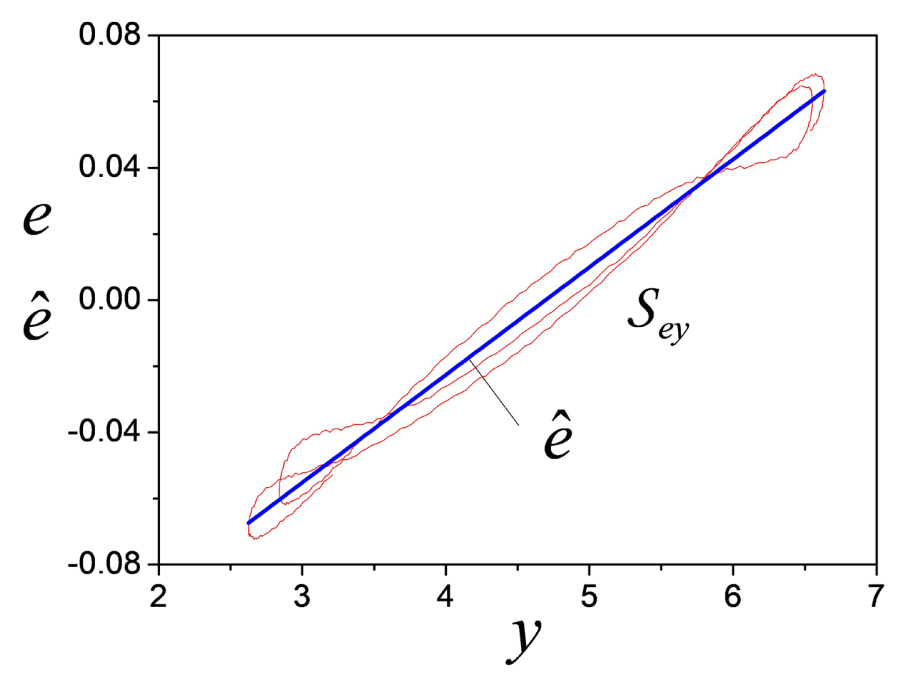

Figure 7. Framework $S_{e y}$.

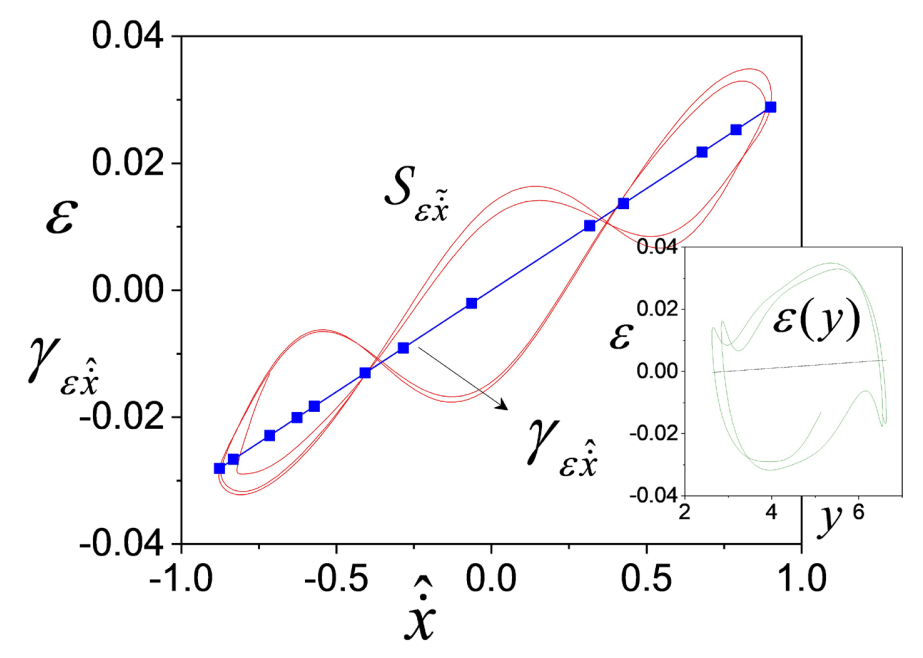

Figure 8. Framework for effect $\hat{\dot{x}}$ estimation of $S_{B W}$-system.

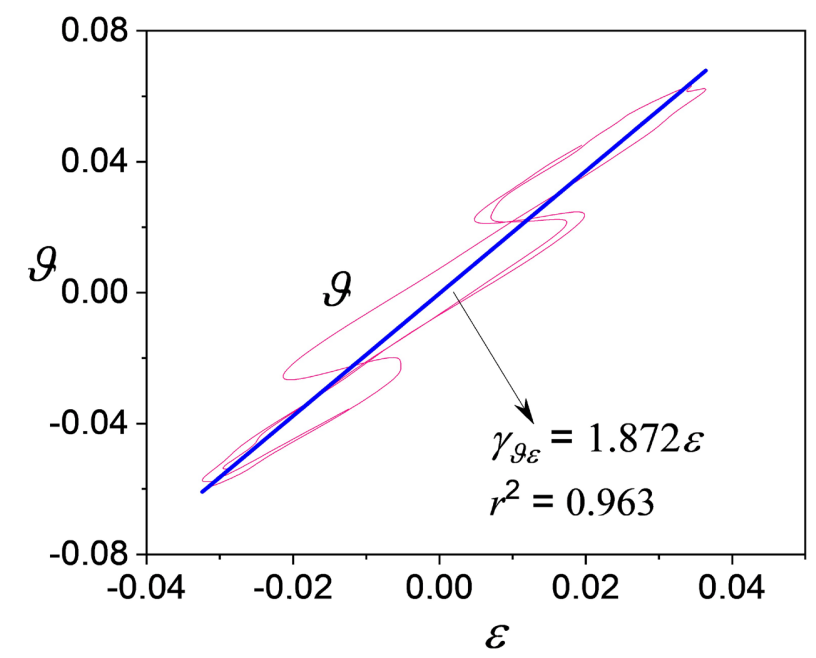

Figure 9. Estimation of correlation $\varepsilon$ and $\vartheta$. 
The effect estimates of the variable $\mu$ can be obtained in space $\mathscr{P}_{\varepsilon \mu}$. This conclusion follows from Figure 9, where the dependency $\vartheta=\vartheta(\varepsilon)$ is presented.

Remark 9. Secant (71) can use as the output for estimating structural relationships in BWM.

Figure 10 confirms the validity of the proposed approach. The framework reflects the relationship between the reference and received hysteresis estimates. The secant $\gamma_{z \hat{z}}$ has the form

$$
\gamma_{z \hat{z}}=0.033 z-0.0068, r_{z \grave{z}}^{2}=0.836 \text {. }
$$

So, the structure analysis has shown that the hysteresis dynamics depends on variables $z$ and $\dot{x}$. The system (1), (2) output does not influence the change of the hysteresis. The structure analysis is based on the application of adequate mathematical methods and guaranteed decision-making on the structure of the system $S_{B W}$.

The HIM stop rule. Let $I_{i}\left(I_{o}\right)$ is an informational set on which the framework $S_{i}$ is defined, where $i$ is the hierarchical immersion level. Examples of sets $I_{i}\left(I_{o}\right)$ and frameworks are presented above. Let $\mathcal{N} S_{i+1}$ is the insignificant framework, and at the level $i$ the system is structurally identifiable.

Let $\mathcal{N} S_{i+1}$ is the insignificant framework, and the system $S_{B W}$ is structurally identifiable at the level $i . i+1$ is a sign of structural non-identifiability the system at the level $i+1 . \mathcal{N} S_{i+1}$ is a sign of the system (1)-(3) structural non-identifiability at level $i+1$.

Theorem 9. The system $S_{B W}$ is structurally identifiable on the set $I_{i}\left(I_{o}\right)$ if $S_{i+1}=\mathcal{N} S_{i+1}$ at the level $i+1$.

The proof of theorem 9 follows from the analysis of secant for framework at each step $i$.

Figure 11 represents the framework $S_{\tilde{\varepsilon} \tilde{\mu}}=\mathcal{N S S}$ and the secant, where $\tilde{\mu}=|\hat{z}||\hat{\dot{x}}|$, $\tilde{\varepsilon}=\vartheta-\gamma_{\vartheta \tilde{\mu}}$. Obtain the model for the variable $\tilde{\varepsilon}$ on the set $\{\hat{\dot{x}}(t), \tilde{\mu}(t)\}$

$$
\hat{\tilde{\varepsilon}}=\hat{a}_{1} \tilde{\mu}+\hat{a}_{2} \hat{\dot{x}},
$$

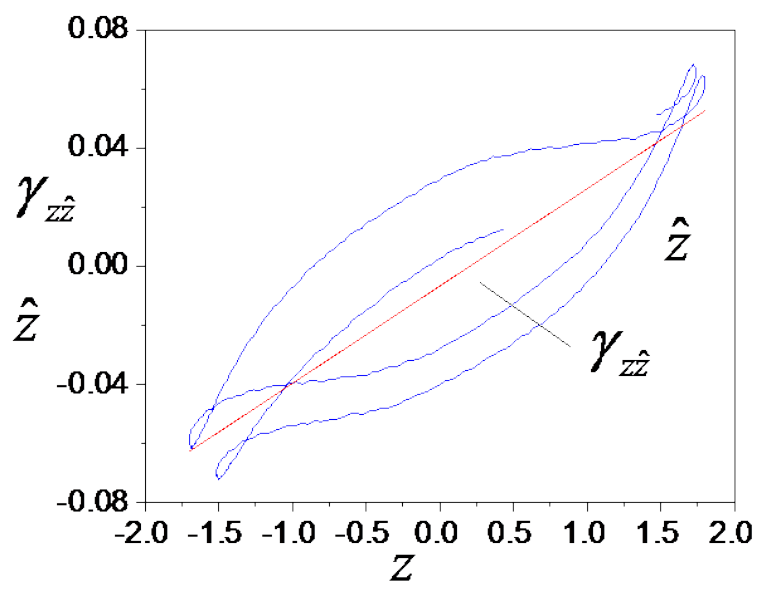

Figure 10. Estimation of proximity $z$ and $\hat{z}$. 


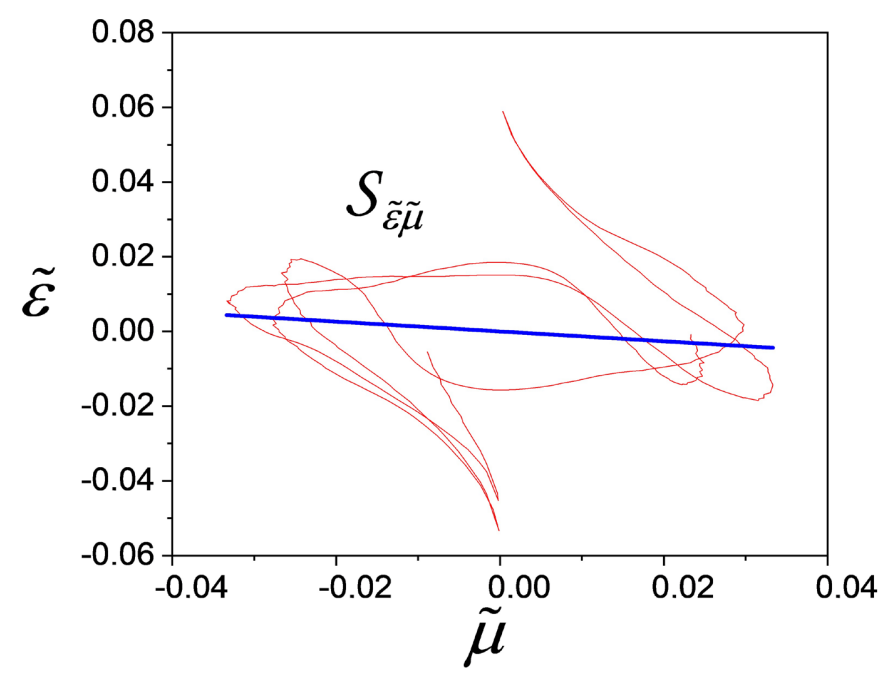

Figure 11. Insignificant framework $S_{\tilde{\varepsilon} \tilde{\mu}}$.

and introduce the misalignment $\tilde{\kappa}=\tilde{\varepsilon}-\tilde{\tilde{\varepsilon}}$. An approximation $\tilde{\kappa}$ by model $\hat{\tilde{\kappa}}=f(\tilde{\mu})$ shows that this relationship is insignificant. This conclusion confirms the presence of the third term in the right part of the Equation (3).

So, we propose the approach for structure estimating of the Bouc-Wen model based on the set $I_{o}$ analysis. The approach is based on the hierarchical immersion method and the analysis of geometrical frameworks. Frameworks describe the state of the system nonlinear part at each SI stage.

\section{Excitation Constancy Effect on System Identifiability}

Let input $u(t)$ of the system (56) have the property

$$
u(t) \in \mathscr{P E} F_{\alpha, \omega_{k}} \text {, }
$$

where

$$
u_{h}(t):\left(u_{h} \in \mathcal{P} E_{\alpha}\right) \&\left(u_{h} \in \mathcal{P} F_{\omega_{h}}\right) \&\left(u_{h} \in S\right), \quad P F_{\omega_{h}}: u_{h}(t)=\mathscr{R} F_{h}\left(\Omega_{h}\right),
$$

$\operatorname{RF}\left(\Omega_{k}\right)$ is a model for $u_{k}(t)$ based on the Fourier series and given on the set of frequencies $\Omega_{k}=\left\{\omega_{1}, \omega_{2}, \cdots, \omega_{k}\right\}$.

Let $u_{k} \in \mathrm{U}_{k}, \mathrm{U}_{k}=\mathrm{U} \backslash \mathrm{U}_{\mathrm{S}}$. Consequently, $u_{k} \notin \mathrm{S}$. For $u_{h} \in \mathrm{S}$ is hold

$$
u_{h}(t):\left(u_{h} \in \mathscr{P} E_{\alpha}\right) \&\left(u_{h} \in \mathscr{P} F_{\omega_{h}}\right) \&\left(u_{h} \in S\right), \quad \mathcal{P F} F_{\omega_{h}}: u_{h}(t)=\mathcal{R} F_{h}\left(\Omega_{h}\right),
$$

where $\Omega_{h} \neq \Omega_{k}$.

Compare (75), (76) and obtain

$$
\left(R F_{h}\left(\Omega_{h}\right) \neq R F_{k}\left(\Omega_{k}\right)\right) \Rightarrow S_{e y}^{h} \neq S_{e y}^{k} \Rightarrow S_{e y}^{k}=\mathcal{N} S_{e y} .
$$

From (77) have

$$
\left(\mathcal{D}_{y}\left(S_{e y}^{h}\right) \neq \mathscr{D}_{y}\left(S_{e y}^{k}\right)\right) \Rightarrow\left[D_{y}\left(S_{e y}^{h}\right) \geq D_{y}\left(S_{e y}^{k}\right)\right] .
$$

The definitional domain of frameworks $S_{e y}^{h}, S_{e y}^{k}$ do not coincide, and $S_{e y}^{h}$ is $d_{h, \Sigma}$-optimal on the set $\mathrm{U}_{h}$. Therefore, the fulfillment of condition (58) follows 
from inequality (78). Consequently, the structure of the system (45) nonlinear part with $u_{k}$ has indicators that do not coincide with the structurally identifiable parameters of the system (45) with $u_{h}$.

So, the CE condition of the input affects the $S_{\varphi}$-system $h_{\delta_{h}}$-identifiable and, consequently, the system (56).

The statement is true.

Theorem 10 [37]. Let (i) the input $u_{k}$ to condition (75); (ii) the $S_{e y}^{k}$ framework corresponds to the input $u_{k}$; (iii) there is the input $u_{h} \in \mathrm{S}$ such that the condition (76) satisfied; (iv) conditions (77), (78) holds. Then (a) the $S_{\varphi}$-system is structurally non-identifiable by the input $u_{k}$; (b) structural parameters of the $S_{\varphi}$-system do not correspond to the system $S_{y \varphi}$ with the identifiable framework $S_{e y}^{h}$.

The input amplitude can influence on the SI of nonlinear systems. Modify conditions (75), (76)

$$
\begin{gathered}
u_{k}(t):\left(u \in \mathscr{P} E_{\alpha}\right) \&\left(u \in \mathscr{P} F_{\omega_{k}}\right) \&\left(\overline{u_{h} \in \mathrm{S}}\right), \quad \mathcal{P} F_{\omega_{k}}: u_{k}(t)=\mathbb{R} F_{k}\left(G_{k}, \Omega_{k}\right), \\
u_{h}(t):\left(u_{h} \in \mathcal{P} E_{\alpha}\right) \&\left(u_{h} \in \mathcal{P} F_{\omega_{h}}\right) \&\left(u_{h} \in \mathrm{S}\right), \quad \mathcal{P} F_{\omega_{h}}: u_{h}(t)=\mathcal{R} F_{h}\left(G_{h}, \Omega_{h}\right),
\end{gathered}
$$

where $G_{k}, G_{h}$ are model $R F_{k}, R F_{h}$ parameter vectors.

Present models $R F_{k}, R F_{h}$ as

$$
R F_{h}\left(G_{h}, \Omega_{h}\right)=g_{h} \widetilde{R F_{h}}\left(\tilde{G}_{h}, \Omega_{h}\right), R F_{k}\left(G_{k}, \Omega_{k}\right)=g_{k} \widetilde{R F_{k}}\left(\tilde{G}_{k}, \Omega_{k}\right),
$$

$\widetilde{R F_{h}}\left(\tilde{G}_{h}, \Omega_{h}\right), \widetilde{R F_{k}}\left(\tilde{G}_{k}, \Omega_{k}\right)$ are modifications of models (78), (76);

$g_{h}=\max _{i} g_{h, i}, i=\overline{1, \# \Omega_{h}}, \quad g_{h, i}$ is an element $G_{h} ; g_{k}=\max _{i} g_{k, i}, i=\overline{1, \# \Omega_{k}}$. $g_{p}(p=k, h)$ denotes the generalized amplitude of the input.

Condition (77) transformed into the form

$$
g_{h} \widetilde{R F_{h}}\left(\tilde{G}_{h}, \Omega_{h}\right) \neq g_{k} \widetilde{R F_{k}}\left(\tilde{G}_{k}, \Omega_{k}\right) .
$$

Since $u_{h} \in \mathrm{S}$ then $g_{h} \geq g_{k}$. This conclusion follows from

$$
D_{h}\left(S\left(u_{h}\right)\right) \geq D_{k}\left(S\left(u_{k}\right)\right) \Rightarrow\left|\widetilde{R F_{h}}\left(\tilde{G}_{h}, \Omega_{h}\right)\right| \geq\left|\widetilde{R F_{k}}\left(\tilde{G}_{k}, \Omega_{k}\right)\right|,
$$

and the model $\widetilde{R F_{h}}\left(\tilde{G}_{h}, \Omega_{h}\right)$ approximates the input ensuring S-synchronization of the system $S_{y \varphi}$.

Obtain $d_{h, \Sigma}$-optimality of the diameter $D_{h}\left(S_{e y}^{h}\right)$ from $s\left(u_{h}\right) \Rightarrow S_{e y}^{h}$. The framework $S_{e y}^{k}$ does not have this property (see (80)). Therefore, the input $u_{k} \notin \mathrm{S}$, which has a smaller generalized amplitude, gives the diameter $D_{k}\left(S_{e y}^{k}\right)$.

Theorem 11 [43]. Let (i) the input $u_{k}$ of the system (45) satisfies the condition (79); (ii) the framework $S_{e y}^{k}$ corresponds to input $u_{k}$; (iii) there is the input $u_{h} \in \mathrm{S}$ such that the condition (80) holds; (iv) conditions (77), (78) are hold. Then (a) the $S_{\varphi}$-system is structurally non-identifiable by the input $u_{k}$; (b) structural parameters of the system $S_{\varphi}$ do not correspond to the system (45) with the identifiable framework $S_{e y}^{h}$ if $g_{h} \geq g_{k}$.

So, the properties influence of input on SI and the structural identifiability of the system with BWH show. 


\section{Conclusion}

The estimate problem of Bouc-Wen hysteresis parameters is relevant under uncertainty. The existing approaches to the identification are based on the parametric paradigm and consider a priori information. Under uncertainty, the BWM synthesis requires time-consuming research. The parametric approach plays an approximative role for a given a priori model structure. It allows you to describe the behaviour of the system or set trends in its development. The structure is a hidden and non-formalized property of the system. Therefore, indirect and object methods should be used that reveal the features (structure) of the system. The paper proposes a structural-identification approach (CIA) for analyzing features of the Bouc-Wen hysteresis under uncertainty. Geometric frameworks are the basis of the CIA. The GF analysis allows for the evaluation of the BWH structure and identifiability. The proposed approaches demonstrate the possibilities of the stated paradigm.

\section{Conflicts of Interest}

The author declares no conflicts of interest regarding the publication of this paper.

\section{References}

[1] Krasnosel'skii, M.A. and Pokrovskii, A.V. (1989) Systems with Hysteresis. Springer-Verlag, Berlin, Heidelberg.

[2] Bouc, R. (1967) Forced Vibrations of a Mechanical System with Hysteresis. Proceedings of the 4th Conference on Nonlinear Oscillations, Prague, 5-9 September 1967, 315-321.

[3] Wen, Y.K. (1976) Method for Random Vibration of Hysteretic Systems. Journal of the Engineering Mechanics Division, 102, 246-263.

[4] Ismail, M. (2009) The Hysteresis Bouc-Wen Model, a Survey. Archives of Computational Methods in Engineering, 16, 161-188.

https://doi.org/10.1007/s11831-009-9031-8

[5] Ismail, M. and Rodellar, J. (2007) Systems with Hysteresis: Analysis, Identification and Control Using the Bouc-Wen Model. Wiley \& Sons Ltd., Hoboken.

[6] Smyth, A.W., Masri, S.F., Kosmatopoulos, E.B., Chassiakos, A.G. and Caughey, T.K. (2007) Development of Adaptive Modeling Techniques for Non-Linear Hysteretic Systems. International Journal of Non-Linear Mechanics, 37, 1435-1451. https://doi.org/10.1016/S0020-7462(02)00031-8

[7] Leenen, R. (2002) The Modelling and Identification of a Hysteretic System: The Wire as a Nonlinear Shock Vibration Isolator. (DCT Reported; 2002.072). Technische Universiteit Eindhoven, Eindhoven.

[8] Chassiakos, G., Masri, S.F., Smyth, A.W. and Caughy, T.K. (1998) Online Identification of Hysteretic Systems. Journal of Applied Mechanics, 65, 194-203. https://doi.org/10.1115/1.2789025

[9] Smith, A.W., Masri, S.F., Chassiakos, A.G. and Caughey, T.K. (1999) Online Parametric Identification of MDOF Nonlinear Hysteretic Systems, Journal of Engineering Mechanics, 125, 133-142. 
https://doi.org/10.1061/(ASCE)0733-9399(1999)125:2(133)

[10] Ioannou, P.A. and Sun, J. (1996) Robust Adaptive Control. Prentice Hall, New York.

[11] Lin, J.W., Betti, R., Smyth, A.W. and Longman, R.W. (2001) On-Line Identification of Nonlinear Hysteretic Structural Systems Using a Variable Trace Approach. Earthquake Engineering and Structural Dynamics, 30, 1279-1303. https://doi.org/10.1002/eqe.63

[12] Shih, M., Sung, W. and Go, C. (2004) Investigation of Newly Developed Added Damping and Stiffness Device with Low Yield Strength Steel. Journal of Zhejiang University—Science A: Applied Physics \& Engineering, 5, 326-334.

[13] Karabutov, N.N. (2019) Identification of System with Bouc-Wen Hysteresis. EPJ Web of Conferences, 224, Article No. 1003.

https://doi.org/10.1051/epjconf/201922401003

https://www.epj-conferences.org/articles/epjconf/abs/2019/29/epjconf_mnps2018_0 1003/epjconf_mnps2018_01003.html

[14] Charalampakis, A.E. and Dimou, C.K. (2010) Identification of Bouc-Wen Hysteretic Systems Using Particle Swarm Optimization. Computers and Structures, 88, 1197-1205. https://doi.org/10.1016/j.compstruc.2010.06.009

[15] Ikhouane, F. and Rodellar, J. (2007) Systems with Hysteresis: Analysis, Identification and Control Using the Bouc-Wen Model. John Wiley \& Sons Ltd., Koboken.

[16] Talatahari, S., Kaveh, A. and Rahbari, N.M. (2012) Parameter Identification of Bouc-Wen Model for MR Fluid Dampers Using Adaptive Charged System Search Optimization. Journal of Mechanical Science and Technology, 26, 2523-2534. https://doi.org/10.1007/s12206-012-0625-y

[17] Nguyen, X.B., Komatsuzaki, T., Hoa, T. and Truong, H. (2021) Adaptive Parameter Identification of Bouc-Wen Hysteresis Model for a Vibration System Using Magnetorheological Elastomer. International Journal of Mechanical Sciences, 213, Article ID: 106848. https://doi.org/10.1016/j.ijmecsci.2021.106848

[18] Danilin, A.N., Kuznetsova, E.L., Kurdumov, N.N., Rabinsky, L.N. and Tarasov, S.S. (2016) A Modified Bouc-Wen Model to Describe the Hysteresis of Non-Stationary Processes. PNRPU Mechanics Bulletin, 4, 187-199.

[19] Ikhouane, F., Hurtado, J.E. and Rodellar, J. (2007) Variation of the Hysteresis Loop with the Bouc-Wen Model Parameters. Nonlinear Dynamics, 48, 361-380. https://doi.org/10.1007/s11071-006-9091-3

[20] Zhang, J. and Sato, T. (2006) Non-Linear Hysteretic Structural Identification by Utilizing On-Line Support Vector Regression. Structural Engineering/Earthquake Engineering, 23, 45s-55s. https://doi.org/10.2208/jsceseee.23.45s

[21] Li, S.J., Suzuki, Y. and Noori, M. (2004) Identification of Hysteretic Systems with Slip Using Bootstrap Filter. Mech. Systems and Signal Processing, 18, 781-795. https://doi.org/10.1016/j.ymssp.2003.08.001

[22] Karabutov, N.N. (2020) Structural Approach to Identification of Nonlinear Manufacturing Systems. In: Mellal, M.A., Ed., Book Manufacturing Systems Recent Progress and Future Directions, Nova Science Publishers, Hauppauge, 121-153.

[23] Karabutov, N. (2018) Structural-Parametrical Design Method of Adaptive Observers for Nonlinear Systems. International Journal of Intelligent Systems and Applications, 10, 1-16. https://doi.org/10.5815/ijisa.2018.02.01

[24] Karabutov, N. (2020) Parameters Adaptive Identification of Bouc-Wen Hysteresis. IFAC-Papersonline, 53, 3971-3976. https://doi.org/10.1016/j.ifacol.2020.12.1339 
[25] Gantmakher, F.R. (1959) Theory of Matrices. Chelsea Pub. Co., New York.

[26] Karabutov, N. (2015) Structural Methods of Estimation Lyapunov Exponents Linear Dynamic System. International Journal of Intelligent Systems and Applications, 7, 1-11. https://doi.org/10.5815/ijisa.2015.10.01

[27] Chang, C.-M., Strano, S. and Terzo, M. (2016) Modelling of Hysteresis in Vibration Control Systems by Means of the Bouc-Wen Model. Shock and Vibration, 2016, Article ID: 3424191. https://doi.org/10.1155/2016/3424191

[28] Fujii, F., Tatebatake, K., Morita, K. and Shiinoki, T. (2018) A Bouc-Wen Model-Based Compensation of the Frequency-Dependent Hysteresis of a Piezoelectric Actuator Exhibiting Odd Harmonic Oscillation. Actuators, 7, Article No. 37. https://doi.org/10.3390/act7030037

[29] Kwok, N., Ha, Q., Nguyen, M., Li, J. and Samali, B. (2007) Bouc-Wen Model Parameter Identification for a MR Fluid Damper Using Computationally Efficient GA. ISA Transactions, 46, 167-179. https://doi.org/10.1016/j.isatra.2006.08.005

[30] Dong, H., Han, Q. and Du, X. (2019) Application of an Extended Bouc-Wen Model for Hysteretic Behaviour of the RC Structure with SCEBs. Structural Engineering and Mechanics, 71, 683-697.

[31] Karabutov, N.N. (2020) Structural Analysis and Modifications of System with Bouc-Wen Hysteresis. Bulletin of the Voronezh State Technical University, 16, 57-64.

[32] Van Pelt, T.H. and Bernstein, D.S. (2001) Nonlinear System Identification Using Hammerstein and Non-Linear Feedback Models with Piecewise Linear Static Maps. International Journal Control, 74, 1807-1823. https://doi.org/10.1080/00207170110089798

[33] Lin, R. and Ewins, D.J. (1995) Location of Localized Stiffness Non-Linearity Using Measured Modal Data. Mechanical Systems and Signal Processing, 9, 329-339. https://doi.org/10.1006/mssp.1995.0027

[34] Trendafilova, I., Lenaerts, V., Kerschen, G., Golinval, J.C. and Van Brussel, H. (2000) Detection, Localization and Identification of Nonlinearities in Structural Dynamics. Proceedings of the International Seminar on Modal Analysis (ISMA), Leuven, 13-15 September 2000, 1-8.

[35] Worden, K. and Tomlinson, G. (2001) Nonlinearity in Structural Dynamics. Detection, Identification and Modelling, Institute of Physics Publishing, Bristol and Philadelphia.

[36] Kerschen, G., Golinval, J.C. and Hemez, F.M. (2003) Bayesian Model Screening for the Identification of Non-Linear Mechanical Structures. Journal of Vibration and Acoustics, 125, 389-397. https://doi.org/10.1115/1.1569947

[37] Karabutov, N. (2015) Structural Identification of Nonlinear Dynamic Systems. International Journal of Intelligent Systems and Applications, 7, 1-11. https://doi.org/10.5815/ijisa.2015.09.01

[38] Kazakov, I.E. and Doctupov, B.G. (1962) Statistical Dynamics of Nonlinear Automatic Systems. Russia Fizmatgiz, Moscow.

[39] Karabutov, N.N. (2011) Structural Identification of Static Plants: Fields, Structures, Methods. URRS/Book House "Librokom", Moscow.

[40] Karabutov, N.N. (2009) Structural Identification of Systems: The Analysis of Informational Structures. URRS/Book House "Librokom", Moscow.

[41] Jauberthie, C., Travé-Massuyès, L. and Verdière, N. (2016) Set-Membership Identifiability of Nonlinear Models and Related Parameter Estimation Properties. International Journal of Applied Mathematics and Computer Science, 26, 803-813. 
https://doi.org/10.1515/amcs-2016-0057

[42] Karabutov, N. (2018) About Structural Identifiability of Nonlinear Dynamic Systems under Uncertainty. Global Journal of Science Frontier Research: A Physics and Space Science, 18, 51-61.

[43] Karabutov N.N. (2020) S-Synchronization Structural Identifiability and Identification of Nonlinear Dynamic Systems. Mekhatronika, Avtomatizatsiya, Upravlenie, 21, 323-336. https://doi.org/10.17587/mau.21.323-336

[44] Karabutov, N.N. (2018) Frameworks in Identification Problems: Design and Analysis. URSS/Lenand, Moscow.

[45] Karabutov, N. (2017) Structural Methods of Design Identification Systems. In: Uvarova, L., Nadykto, A.B. and Latyshev, A.V., Ed., Nonlinearity: Problems, Solutions and Applications. Vol. 1, Nova Science Publishers Inc., Hauppauge, 233-274. 